\title{
Direct Simulation on Nonlinear Thermokinetic Phenomena Due to Induced-Charge Electroosmosis
}

\author{
Hideyuki Sugioka ${ }^{1} \dagger$ \\ ${ }^{1}$ Department of Mechanical Systems Engineering, Shinshu University 4-17-1 Wakasato, \\ Nagano 380-8553, Japan
}

(Received xx; revised xx; accepted xx)

Previously, we proposed a novel mechanism to produce nonlinear thermokinetic phenomenon (NTKP) around a metal cylinder in an electrolyte on the basis of analytical discussion. In this study, by using a nonsteady direct multi-physics simulation technique based on the Stokes equation coupled with the electroosmotic equation that considers normal diffusion, electrophoresis, and thermal diffusion, we directly verify the NTKP and show that the original driving force is the excess ions pressed on the particle by the thermokinetic force and the NTKP vortex flow around the particle is generated by the interaction between the excess ion and the electric field that is made by the excess ion itself and/or the Seebeck electric field due to the blocking boundary condition on the wall. Namely, two types of NTKP exist and they are explained in a self-consistent manner by our new theory. In addition, through the discussion on a dielectric particle, we show that NTKP is a general phenomenon that can be found in both metal and dielectric particles. We believe that our findings provide a new unified viewpoint to understand complex thermokinetic phenomena near metal and dielectric particles.

Key words: nonliniear thermokinetic phenomena, induced charge electroosmosis, Seebeck effect, thermoelectric effect

\section{Introduction}

The nonequilibrium thermokinetic problem (in liquid) that includes electrokinetic phenomena usually becomes an extremely challenging problem. What makes the problem difficult? One main reason is the lack of the cross-sectional integrated physical concept, except the fact that the nonequilibrium thermodynamics has not been completely established yet, although many challenging work devoted for this purpose (Würger 2010). The thermokinetic phenomena have been known as the Soret effect since 19th century and they are recognized as important phenomena, which can be used for various biomedical applications, such as an efficient separation technique for macro molecules (Giddings 1993) and a molecular trap for DNA (Duhr \& Braun 2006a). That is, under the existence of a temperature gradient $\nabla T$, the thermal drift velocity is described as

$$
\boldsymbol{u}=-D_{T} \nabla T
$$

$\dagger$ Email address for correspondence: hsugioka@shinshu-u.ac.jp 
based on the Onsager's reciprocal theorem with the definition of a Soret coefficient

$$
S_{T} \equiv D_{T} / D,
$$

where $D$ is the ordinary diffusion coefficient of Einstein and $D_{T}$ is the thermo diffusion coefficient. Here, $D_{T}$ and $S_{T}$ are generally considered just as a phenomenological parameter determined by experiments in spite of many theoretical efforts; i.e., the generally accepted complete theory that accounts for the puzzling behaviors of $D_{T}$ (or $S_{T}$ ) for all situations does not exist and many unsolved problems remain in this field (Huang et al. 2010; Würger 2010, 2008, 2007; Eslamian \& Saghir 2009). Note that the difficulty exists in the remarkable sensitivity of $S_{T}$ (and $D_{T}$ ) to many factors (Huang et al. 2010); e.g., a size (Duhr \& Braun 2006b) and temperature (Putnam et al. 2007). This is why this field is challenging.

It is natural to consider the extended Nernst-Plank (NP) equations that consider the thermokinetic term using a Eastman entropy of transfer $Q^{*} / T$ (Eastman 1928, 1926) as a fundamental equation at least for the colloidal particle that has an electric double layer, where $Q^{*}$ is a ionic heat of transfer and it is studied extensively by Arger et al. (Agar et al. 1989). Further, although researchers in the field of thermokinetic study often neglect a electrokinetic term, some researchers pointed out the importance of the electrokinetic term and the effect is specially recognized as the thermoelectric effect from long ago (Gutherie et al. 1949; Putnam et al. 2007; Würger 2010). For example, Guthrie et al. succeeded in explaining the anomalous difference between the thermal diffusion coefficient of an ion in the presence and in the absence of other electrolyte based on the equation that considers the molecular diffusion, thermal diffusion, and electrophoretic terms in 1949 (Gutherie et al. 1949). Putnam and Cahill (Putnam \& Cahill 2005) showed that $D_{T}$ of charged latex spheres in aqueous suspensions is controlled by the Seebeck coefficient of the electrolyte $\left(S_{e}\right)$ in 2005. Würger (Würger 2008) showed that $D_{T}$ of charged colloids is determined by the thermoelectric effect to the large extent by using explicitly the extended NP equation that comprises a thermodiffusion term described by the Eastman entropy as a fundamental equation for the system in 2008 . Furthermore, Majee and Wüger (Majee \& Würger 2012) predicted charging phenomena of heated colloidal particles based on the extended NP equation along with the electrolyte or fluidic Seebeck effect. Thus, in this manuscript, we also use the extended NP equation as the fundamental equation toward a step of a general design base that bridges both electrokinetic and thermokinetic studies.

The study on the fluidic Seebeck effect is usually based on the extended NP equation (Bonetti et al. 2011; Agar et al. 1989; Majee \& Würger 2012); i.e., the fluidic Seebeck effect is defined as a thermoelectric effect that occurs in liquid and it is often characterized by the Seebeck coefficient $S_{e}$ derived by the zero current condition for the extended NP equation (Bonetti et al. 2011; Würger 2008; Majee \& Würger 2012). The fluidic Seebeck effect has also attracted much attention because of the potentiality for an innovative thermogalvanic cell. For example, Bonetti et al. showed a huge Seebeck effect in nonaqueous electrolyte Bonetti et al. (2011). Hu et al. demonstrated a high-performance thermogalvanic cell using a carbon-nanotube (Hu et al. 2010). However, the importance of the boundary conditions and the distribution of ions have been just recently recognized and investigated by Chikina et al. (Chikina et al. 2012, 2015), as a pioneering work. Thus, extensive researches for the thermokinetic boundary conditions are highly required.

Just recently, a metal capped insulative particle called a Janus particle has attracted much attention from the view point of self-propulsion mechanism and possible applications such as microswimmers and nanomachines. For example, Jiang et al. (Jiang et al. 2010) experimentally showed that the Janus particle irradiated by the laser can move in 
the direction of the dielectric end in water by thermophoresis due to the local temperature gradient in a particle. Bickel et al. (Bickel et al. 2013) theoretically showed the flow around the metal capped-Janus particle by assuming a thermophoretic surface-slip velocity proportional to the temperature gradient. However, the polarizable characteristics of the self-propelled Janus particle has not been considered yet in spite of its importance. In particular, the experimental thermokinetic velocity of the self-propelled Janus particle $u_{J}$ (Jiang et al. 2010) does not proportional to $\nabla T$ globally in the sense that the line of the dependence $\left(u_{J}\right.$ vs. $\left.\nabla T\right)$ does not go through zero point. Thus, the thermokinetic phenomena in the vicinity of a polarizable material should be explored.

The electrokinetic phenomena in the vicinity of a polarizable material such as a metal particle and an electrode have just recently been recognized (Ramos et al. 1999, 2003; Bazant \& Squires 2004; Bazant et al. 2009) and the new knowledge forces us to reconsider many fundamental issues on surface science in electrolyte with the expectation for the tremendous microfluidic applications (Squires 2009; Sugioka 2015b), although the study of electrokinetic phenomena often considered to be matured. Namely, an electrokinetic vortex flow due to the interaction between a charge in an electric double layer and a tangential electric fields near the surface of planar electrodes has first recognized as ac electroosmosis (ACEO) in 1999 (Ramos et al. 1999). Promptly, the phenomena are recognized as more general phenomena near a polarizable material and termed as induced-charge electroosmosis (ICEO) in 2004 (Bazant \& Squires 2004). Here, ICEO includes ACEO and it results from the general interaction between the electric field and charges in the electric double layer formed by the polarizing effect. In particular, ICEO is typically observed as a quadrupolar vortex flow around the conductive cylinder in electrolyte subject to an external electric field and it is characterized by the flow velocity proportional to the square of the applied electric field; i.e., ICEO is a nonlinear electrokinetic phenomenon. Various microfluidic applications such as pumps, valves, mixers, self-propelled Janus particle (the particle containing metallic and insulative surfaces), etc. (Bazant \& Squires 2004; Sugioka 2010, 2015a) have been proposed because of its large flow velocity $(\sim 1 \mathrm{~mm} / \mathrm{s})$ at small voltages $(\sim 1 \mathrm{~V})$ and examined experimentally (Urbanski et al. 2006; Gangwal et al. 2008; Pascall \& Squires 2010) in this decade; and they have attracted much attention as the promising leadingedge technologies in microfluidics. However, even now, the characteristics of ICEO have not been clarified completely and the challenging study trying to establish a design base for ICEO devices has been continued extensively (Bazant et al. 2009; Squires 2009; Sugioka 2015b, 2016). In particular, ICEO under the existence of a temperature gradient has not been discussed well except our previous study (Sugioka 2014b), although a large temperature gradient often exists in an microfluidic systems because of the heat flow from an electric integrated circuit of driver or the use of heating devices in a channel. Thus, the study on ICEO under the large temperature gradient is important.

In the previous study (Sugioka 2014b), we proposed a novel mechanism to produce nonlinear thermokinetic phenomena around a metal cylinder under a large temperature gradient on the basis of analytical discussion. The most characteristic aspect of the nonlinear thermokinetic phenomena is the generation of quadrupolar vortex flows, which flow velocity is proportional to the square of the temperature gradient [i.e., $|\boldsymbol{u}| \propto$ $\left.(\nabla T)^{2}\right]$, and the Janus particle using this phenomenon can move to the both hotter and colder regions in a temperature gradient by changing the direction of its dielectric end (Sugioka 2014b). However, since the nonlinear thermokinetic phenomena are very complex phenomena related to the fluidic Seebeck effect and ICEO, the details of the nonlinear thermokinetic phenomenon (NTKP) has not been explored yet. In particular, the nonsteady behavior of ions near metal under the large temperature gradient has not 


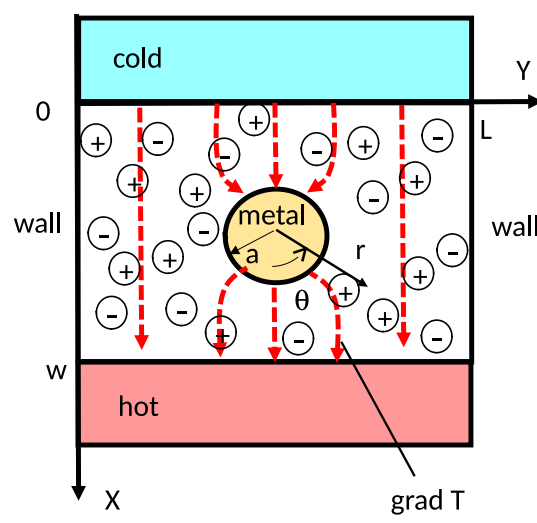

(a) Initial state

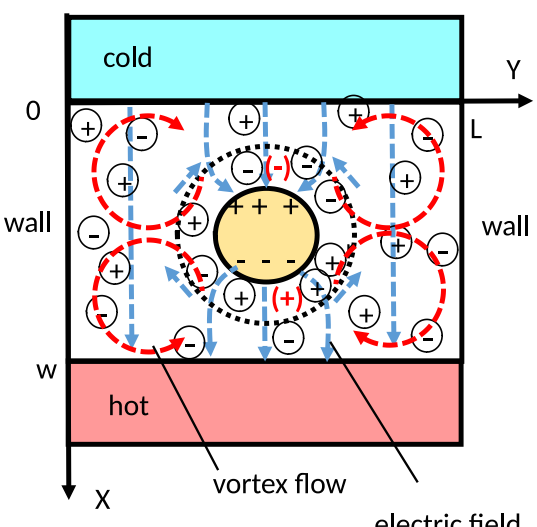

(b) Steady state

Figure 1. (Color online) Primitive mechanism and considered system of nonlinear themokinetic phenomenon (NTKP) on our direct simulation. Here, $(\nabla T)_{a}(=1 \mathrm{~K} / \mu \mathrm{m})$ is an externally applied temperature gradient, $a=0.1 W$ is a radius of a metal cylinder, and $W(=50 \mu \mathrm{m})$ is the distance between the hot and cold boundaries.

been explored yet, although the pioneering study on the linear thermokinetic phenomena on metal exists (Giddings et al. 1995). On the other hand, by several groups (Gregersen et al. 2009; Davidson et al. 2014; Sugioka 2014a, 2016), direct calculation methods that calculate ICEO flows directly based on the governing equations without using a thin double layer approximation are developed, although the direct calculation method that can calculate both the fluidic Seebeck effect and ICEO has not been reported yet to the best of my knowledge.

Therefore, in this paper, we focus on the nonsteady direct simulation around a metal cylinder under the large temperature gradient and fundamentally clarify the nonlinear thermokinetic phenomena due to the thermoelectric effect and ICEO. In particular, by using the 2D nonsteady direct simulation technique that considers the modified NernstPlanck (NP) equation that comprises thermophoretic diffusion term with the Stokes and Poisson equations, we will show that the quadrupolar vortex flows due to the nonlinear thermokinetic phenomena are naturally generated and become the steady state.

This paper is presented in five sections. In Sec. II, we describe the theories for a geometry model, a primitive mechanism, a numerical method, the dimensionless formulations, the considered modes, and the simple analytical model. Based on these theories, the results for the basic nonsteady analysis for various modes are presented in Sec. III. Following a discussion in Sec. IV, our conclusions are summarized in Sec. V. 


\section{Theory}

\subsection{Geometry model}

In Fig. 1, we consider a system having a metal cylinder of radius $a=0.1 \mathrm{~W}$ at the center position $(x, y)=(X / W, Y / W)=(0.5,0.75)$ in an electrolyte (typically water) between hot and cold parallel plates, which distance and length are $W(=50$ $\mu \mathrm{m})$ and $L(=1.5 \mathrm{~W})$, respectively. Since the thermal conductivity of a metal $\lambda_{m}$ is usually much larger than that of the surrounding electrolyte $\lambda_{e}$, we can assume that $\lambda_{m}=\infty$. Furthermore, since the thermal diffusivity (of water) is also much larger than the molecular diffusivity, the temperature distribution becomes a steady state much faster than the ion distribution. Thus, a temperature around the metal cylinder can be approximated analytically (Sugioka 2014b) as

$$
T \simeq T_{a}+\left(1-\frac{a^{2}}{r^{2}}\right)(\nabla T)_{0} r \cos \theta
$$

on the basis of the solution of the steady heat diffusion equation $\nabla^{2} T=0$ for an unbounded problem, where $T_{a}(=300 \mathrm{~K})$ is the temperature of the cylinder, $r$ is the radial coordinate, and $\theta$ is the angular coordinate. Note that the effectiveness of Eq. (2.1) is also examined through our simulations.

\subsection{Primitive mechanism}

At the temperature of Eq. (2.1), the positive and negative ions (e.g., $\mathrm{H}^{+}$and $\mathrm{Cl}^{-}$) move in the direction of a colder region along to the heat flux, as shown in Fig. 1(a). However, because of the difference of the thermal diffusivity of the ions, net negative (positive) charge is generated in the colder (hotter) region of the metal cylinder. Thus, the counter charges are induced on the surface of the metal cylinder and form an electric double layer. Note that although the induced charge cancels the electric field in the diffused ion region, the tangential electric field remains. Thus, quadrupolar vortex flows are generated around the metal cylinder as shown in Fig. 1(b). Here, we neglects the convection flow due to the difference of the density as a first step since it is usually suppressed in a thermophoretic measurement.

\subsection{Governing equations}

We consider the Poisson equation, the extended Nernst-Planck (NP) equation including the thermokinetic diffusion term with the advection flow term, the diffusion-advection equation for the temperature field, and the Stokes equation as follows:

$$
\begin{array}{r}
\varepsilon \nabla^{2} \Phi+\rho=0, \\
\frac{\partial C_{ \pm}}{\partial t}=-\nabla \cdot\left[-D_{ \pm}\left(\nabla C_{ \pm} \pm \frac{z e}{k T} C_{ \pm} \nabla \Phi+\frac{Q_{ \pm}}{k T} C_{ \pm} \frac{\nabla T}{T}\right)+C_{ \pm} \boldsymbol{u}\right], \\
\frac{\partial T}{\partial t}=-\nabla \cdot\left(-a_{T} \nabla T+T \boldsymbol{u}\right), \\
-\nabla P+\mu \nabla^{2} \boldsymbol{u}-\rho \nabla \Phi=0, \quad \nabla \cdot \boldsymbol{u}=0,
\end{array}
$$

where $\varepsilon=80 \varepsilon_{0}$ is the dielectric permittivity of the solvent (typically water), $\varepsilon_{0}$ is the vacuum permittivity, $t$ is time, $\boldsymbol{u}_{d}\left[=\left(u_{x}, u_{y}\right)\right]$ is the flow velocity, $\Phi$ is the potential, $\mu$ $(\sim 1 \mathrm{mPa} \cdot \mathrm{s})$ is the viscosity, $k$ is the Boltzmann constant, $P$ is the pressure, $z e$ is the ion charge, and $\rho\left[=z e\left(C_{+}-C_{-}\right)\right]$is the charge density. Furthermore, $C_{ \pm}, D_{ \pm}$, and $Q_{ \pm}$are the ion concentration, the ion diffusivity, and the ionic heat of transport, respectively, of the positive and negative ions; $a_{T}\left(=\lambda / \rho_{m} c\right)=148 \times 10^{-9} \mathrm{~m}^{2} / \mathrm{s}$ for water $)$ is the thermal 


$\begin{array}{cccc}\text { Mode } & \text { B.C. } & \text { T } & \text { T dependence } \\ 1 & \text { passing } & \text { steady } & \text { no } \\ 2 & \text { passing } & \text { nonsteady } & \text { no } \\ 3 & \text { passing } & \text { nonsteady } & \text { yes } \\ 4 & \text { blocking } & \text { steady } & \text { no } \\ 5 & \text { blocking } & \text { nonsteady } & \text { no } \\ 6 & \text { blocking } & \text { nonsteady } & \text { yes }\end{array}$

TABLE 1. List of modes.

diffusivity, $\lambda$ is the thermal conductivity, $c$ is the specific heat, and $\rho_{m}$ is the density of electrolyte.

\subsection{Dimensionless formulations}

It is useful to consider dimensionless formulations corresponding to Eqs. (2.2)-(2.5) as follows:

$$
\begin{array}{r}
2 \epsilon^{2} \nabla_{n}^{2} \phi+\rho_{n}=0 \\
\frac{\partial c_{ \pm}}{\partial \tau}=-\nabla_{n} \cdot \boldsymbol{f}_{ \pm}, \\
\boldsymbol{f}_{ \pm}=-\tilde{D}_{ \pm}\left(\nabla_{n} c_{ \pm} \pm c_{ \pm} \frac{\nabla_{n} \phi}{\tilde{T}}+2 \frac{\alpha_{ \pm}}{\tilde{T}} c_{ \pm} \frac{\nabla_{n} \tilde{T}}{\tilde{T}}\right)+c_{ \pm} \boldsymbol{u}_{n} \\
\frac{\partial \tilde{T}}{\partial \tau}=-\nabla_{n} \cdot\left(-\frac{a_{T}}{D_{0}} \nabla_{n} \tilde{T}+\tilde{T} \boldsymbol{u}\right), \\
-\nabla_{n} p+\tilde{\mu} \nabla_{n}^{2} \boldsymbol{u}_{n}-F_{0} \rho_{n} \nabla_{n} \phi=0, \quad \nabla_{n} \cdot \boldsymbol{u}_{n}=0,
\end{array}
$$

where $\boldsymbol{u}_{n}\left(=\boldsymbol{u} / u_{c}\right), \phi\left(=\Phi / \Phi_{c}\right), c_{ \pm}\left(=C_{ \pm} / C_{0}\right), \rho_{n}\left(=\rho / \rho_{c}=c_{+}-c_{-}\right), \tau\left(=t / T_{0}\right)$, $p\left(=P / P_{c}\right), \tilde{T}\left(=T / T_{a}\right), \tilde{\mu}\left(=\mu / \mu_{0}\right), \tilde{D_{ \pm}}\left(=D_{ \pm} / D_{0}\right)$, and $(x, y)[=(x / W, Y / W)]$ are the nondimensional values of a flow velocity, potential, concentration, charge density, time, pressure, temperature, viscosity, diffusivity, and position, respectively. Further, $\nabla_{n}=\nabla / W, \epsilon=\lambda_{D} / W, \boldsymbol{f}_{+}\left(\boldsymbol{f}_{-}\right)$is a nondimensional flux of a positive (negative) ion, $\lambda_{D}\left(\equiv \sqrt{\varepsilon k T_{a} / 2 z^{2} e^{2} C_{0}}\right)$ denotes the Debye length, $T_{0}\left(=W^{2} / D_{0}\right)$ (typically, $2.5 \mathrm{~s}$ ) is a diffusion time, $F_{0}=k T_{a} C_{0} T_{0} / \mu_{0}$ is a nondimensional number related to the strength of a Coulomb force; $T_{a}(300 \mathrm{~K}), \mu_{0}(1 \mathrm{mPa} \mathrm{s}), D_{0}\left(10^{-9} \mathrm{~m}^{2} / \mathrm{s}\right), u_{c}=W / T_{0}$ (typically, $20 \mu \mathrm{m} / \mathrm{s}), P_{c}=\mu_{0} / T_{0}, \Phi_{c}=k T_{a} / z e$, and $\rho_{c}=z e C_{0}$ are the reference values of the temperature, viscosity, diffusivity, flow velocity, pressure, potential, and charge density, respectively. Furthermore, we define $\alpha_{ \pm}$as follows:

$$
\alpha_{ \pm}=\frac{Q_{ \pm}}{2 k T_{a}}
$$

\subsection{Considered modes (Modes 1 to 6 )}

\subsubsection{Boundary conditions}

We summarize considered modes in Table. I. We assume a passing boundary condition at the edges for Modes 1 to 3, whereas we assume a blocking boundary condition for Modes 4 to 6 ; i.e., at $x=0$ and 1 ,

$$
\begin{aligned}
& \boldsymbol{f}_{ \pm} \neq 0 \text { (for passing problems), } \\
& \boldsymbol{f}_{ \pm}=0 \text { (for blocking problems). }
\end{aligned}
$$


Here, the ion passing condition means that ions at the colder edge (i.e., $x=0$ ) can diffuse to the hotter edge (i.e., $x=1$ ) promptly through an external electrical circuit; i.e., we use a kind of periodic boundary condition for ion transportation and we assume an artificial flux (Sugioka 2014a) to expel the ion near the edges as

$$
\boldsymbol{f}_{ \pm}=-\overline{\tilde{D_{ \pm}}} \frac{c_{ \pm}^{x=\Delta x / 2}-c_{ \pm}^{1-\Delta x / 2}}{x_{m}} \boldsymbol{i},
$$

where $\boldsymbol{i}$ is a unit vector in the direction of $x, \Delta x_{m}$ is a $x$ width of the upper and lower cells next to the upper and lower edges in the FVM for the NP equation, and $\overline{\tilde{D}}_{ \pm}\left[=\left(D_{ \pm}^{x=0}+D_{ \pm}^{x=1}\right) / 2\right]$ is the average ion diffusivity at the edges. Physically, the ion passing condition has various means as discussed in the Appendix C of Ref. (Sugioka 2014a); e.g., it is corresponding to an experiment using an idealized thermogalvanic cell, in which the chemical reactions are assumed to occur at the edges. However, we here use it just as the better approximation for the unbounded problem, since the chemical reactions due to thermophoresis is very complex phenomena and it is beyond the scope of this manuscript.

Further, the ion blocking condition means that ions next to the assumed wall at the edges can not pass them and physically it is corresponding to a thermophoretic experiment using a glass chamber or the open electrodes that does not go through Faraday current. Note that we do not consider a zero-electric-field boundary condition that is often used at a wall in a glass chamber (Chikina et al. 2012, 2015; Majee \& Würger 2012), since it is more artificial than our method in which ions just can not go through the wall at the edges. In addition, we also assume the ion blocking condition on the metal cylinder for all the modes; i.e., $\boldsymbol{f}_{ \pm}=0$ at $\tilde{r}=0.1$. Further, we assume that the flow velocity is zero at the left and right walls, the upper and lower plates, and the surface of the metal cylinder; i.e., $\boldsymbol{u}_{n}=0$ at $x=0$ and $1, y=0$ and 1.5, and $\tilde{r}=0.1$. Furthermore, for simplicity, we assume that $\Phi=0$ on the surface of the metal cylinder.

\subsubsection{Calculation method of a temperature}

We assume Eq. (2.1) for Modes 1 and 4, whereas we consider the nonsteady temperature obtained by Eq. (2.9) under the condition that $\tilde{T}(x, y)=1$ at $\tau=0$ for Modes 2, 3, 5, and 6. In Eq. (2.9), $a_{T} / D_{0}$ (typically, 148 for water) is the nondimensional number related to thermal and ion diffusivities; since $a_{T} / D_{0} \gg 1$, we can assume Eq. (2.1) if we are not interested in the nonsteady effect. Note that the nondimensional form of Eq. (2.1) is

$$
\tilde{T} \simeq 1+\left(1-\frac{\tilde{a}^{2}}{\tilde{r}^{2}}\right)\left(\nabla_{n} \tilde{T}\right)_{0} \tilde{r} \cos \theta
$$

where $\tilde{a}=\frac{a}{W}, \tilde{r}=\frac{r}{W}$, and $\left(\nabla_{n} \tilde{T}\right)_{0}=\left(\nabla_{n} T\right)_{0} \frac{W}{T_{a}}$ [ typically, $(1 \mathrm{~K} / 1 \mu \mathrm{m}) \times(50 \mu \mathrm{m} / 300 \mathrm{~K})$ $=0.167]$. Since the initial temperature distribution $[\tilde{T}(x, y)=1$ or Eq. (2.15)] may affect the result of a flow velocity in a steady state, we examine the effect in this manuscript.

\subsubsection{Temperature dependence of the parameters}

The temperature around the metal cylinder is $T_{a}$ and our concerned phenomena can be generated in the electric double layer of the thickness of $\lambda_{D}(1 \mu \mathrm{m}$, through this manuscript); i.e., the temperature difference of a concerned region is just about $2 \mathrm{~K}$. Thus, the effects of the temperature dependence is assumed to be small. Nevertheless, one may criticize that the variation of the coefficients of viscosity $\mu$, electrophoretic and thermophoretic mobility, and ion diffusivity can not be negligible. Thus, we examine the effects in this manuscript. However, it is not good idea to consider the complex temper- 
ature dependence of all parameters from the beginning since we cannot recognize the main effects of our system because of the complexity. Thus, we assume the temperature dependence only for Modes 3 and 6.

Specifically, for a dilute $\mathrm{HCl}$ solution, we assume as follows:

$$
\begin{array}{r}
\tilde{\mu}=\tilde{a}_{\mu} \exp \left(\frac{\tilde{b}_{\mu}}{\tilde{T}}\right), \\
\tilde{D_{ \pm}}=\tilde{a}_{ \pm} \exp \left(\frac{-\tilde{b}_{ \pm}}{\tilde{T}}\right),
\end{array}
$$

where Eq. (2.16) is known as the Andrade equation (E. N. da C. Andrade 1952) and the parameters are determined by the well-known data for water (JSME 2009) as $\tilde{a}_{\mu}=1.369$ and $\tilde{b}_{\mu}=6.475$; Eq. (2.17) is known as the Arrhenius equation and the parameters are determined by the data in Ref. (Li \& Gregory 1974) as $\tilde{a}_{+}=2358$ and $\tilde{b}_{+}=5.500$ for a $\mathrm{H}^{+}$ion, and $\tilde{a}_{-}=4169$ and $\tilde{b}_{-}=7.580$ for a $\mathrm{Cl}^{-}$ion. Further, electroosmotic mobilities for positive and negative ions are described by the Einstein relation as

$$
\kappa_{e, \pm}=\frac{D_{ \pm}}{k T}
$$

and it is already considered in Eq. (2.3). Thus, by considering Eq. (2.17), the temperature dependence of $\kappa_{e, \pm}$ are considered completely. Furthermore, thermoosmotic mobilities for positive and negative ions are described as

$$
\kappa_{T, \pm}=\kappa_{e, \pm} S_{ \pm}=\frac{D_{ \pm}}{k T} \frac{Q_{ \pm}}{T}
$$

and it is already considered in Eq. (2.3). Here, $S_{ \pm}=\frac{Q_{ \pm}}{T}$ is the Eastman entropy for positive and negative ions. Thus, by considering Eqs. (2.16) and (2.17), the temperature dependence of $\kappa_{T, \pm}$ are considered almost completely. Note that the Eastman's heat of transfer is often measured under the condition of temperature difference of $20 \mathrm{~K}$ (by Paul Delahay 1963) and thus the temperature dependence of $Q_{ \pm}$is usually considered to be neglected.

\subsection{Numerical method using a multi-physics simulation technique}

We use the finite volume method (FVM) for the NP part and the heat conduction part to assure the ion conserving condition, whereas we use the finite element method (FEM) for the parts of the Poisson and Stokes equations to assure the accuracy of the calculations; i.e., our calculations are performed in the framework of the FVM-FEM coupling method described in (Sugioka 2014a). Specifically, we first generate unstructured meshes around the metal cylinder and structured meshes in the far positions from the cylinder; then, for the completely nonsteady calculations (Modes 2, 3, 5, and 6 in Table 1), we solve the heat conduction equation of Eq. (2.9) by the FVM, the extended NP equation of Eq. (2.7) by the FVM, the Poisson equation of Eq. (2.6) by the FEM, and the Stokes equation of Eq. (2.10) by the FEM, iteratively. Further, for the incompletely nonsteady calculations (Modes 1 and 4 in Table 1), we use the solution of Eq. (2.15) without solving Eq. (2.9). Note that the most of the algorism of our calculations is the same as that described in Appendix A in Ref. (Sugioka 2014a) except it considers thermophoretic motions of ions. Thus, here, we briefly describe the main different part; i.e., since the difference of $\tilde{D}_{+}$and $\tilde{D}_{-}$is intrinsic for the nonequilibrium thermokinetic study, we consider the discretized matrix formulation of the PN part [Eqs. (2.7) and 
(2.8)] for the FVM as follows:

$$
\begin{gathered}
\left(1+\sum_{j}^{j=4} \tilde{D}_{ \pm, i j} G_{i j}^{A}\right) c_{ \pm}^{i, n+1}-\sum_{j}^{j=4} \tilde{D}_{ \pm, i j} G_{i j}^{A} c_{ \pm}^{j, n}=c_{ \pm}^{i, n} \mp \sum_{j}^{j=4} \frac{\tilde{D}_{ \pm, i j}}{\tilde{T}^{i j, n}} G_{i j}^{A} c_{ \pm, i j}^{n+1} \delta \phi^{i j, n} \\
-\sum_{j}^{j=4} G_{i j}^{B} c_{ \pm, i j}^{n} \boldsymbol{u}_{i j}^{n} \cdot \mathbf{n}_{i j}-\sum_{j}^{j=4} \frac{2 \tilde{D}_{ \pm, i j} \alpha_{ \pm}}{\left(\tilde{T}^{i j, n}\right)^{2}} G_{i j}^{A} c_{ \pm, i j}^{n} \delta \tilde{T}^{i j, n}
\end{gathered}
$$

where $\delta \phi^{i j, n}=\phi^{i, n}-\phi^{j, n}, \delta \tilde{T}^{i j, n}=\tilde{T}^{i, n}-\tilde{T}^{j, n}, \tilde{T}^{i j, n}=\frac{\tilde{T}^{i, n}+\tilde{T}^{j, n}}{2}, G_{i j}^{A}=\frac{\Delta t S_{i j}}{V_{i} d_{i j}}\left(\boldsymbol{j}_{i j} \cdot \mathbf{n}_{i j}\right)$, and $G_{i j}^{B}=\frac{\Delta t S_{i j}}{V_{i}}$. Here, $V_{i}$ is a volume of the i-th finite element at $\boldsymbol{x}_{i}, S_{i j}$ is the boundary area, and $\boldsymbol{n}_{i j}$ is the outward unit vector normal to $S_{i j}$. Further, $\mathbf{u}_{i j}^{n}$ is the velocity between $i$ 'th and $j$ 'th cells, $c_{ \pm, i j}^{n}=\left(c_{ \pm}^{i, n}+c_{ \pm}^{j, n}\right) / 2, d_{i j}$ is a distance between the $i$ 'th and $j$ 'th center points, $\phi^{i, n+1}\left(=\frac{1}{4} \sum_{\beta=1}^{4} \phi_{\beta}\right)$ is a center value of $\phi$ at $i$ 'th cell, $\boldsymbol{j}_{i j}=\left(\boldsymbol{x}^{j}-\boldsymbol{x}^{i}\right) /\left|\boldsymbol{x}^{j}-\boldsymbol{x}^{i}\right|$, and the suffixes $n$ and $n+1$ show the value at $t=t_{n}$ an $t_{n+1}$, respectively.

\subsection{Simple analytical theory}

We consider a simple analytical theory based on Ref. (Sugioka 2014b) as follows:

$$
\begin{array}{r}
u^{\max }=f u_{n t k p}, \\
u_{n t k p}=\frac{\varepsilon a}{\mu} E_{n t k p}^{2}, \\
E_{n t k p}=S_{e}(\nabla T)_{0} \\
S_{e}=\frac{D_{+} \alpha_{+}-D_{-} \alpha_{-}}{D_{+}+D_{-}} \frac{2 k}{e Z}
\end{array}
$$

where $S_{e}$ is a Seebeck coefficient (e.g., $S_{e}=374 \mu \mathrm{V} / \mathrm{K}$ for a $\mathrm{HCl}$ solution); $u_{n t k p}$ and $E_{n t k p}$ are the theoretical maximum flow velocity and electric field predicted for the unbounded problem (Sugioka 2014b) of the nonlinear thermokinetic phenomena; e.g., $u_{n t k p}=0.495 \mu \mathrm{m} / \mathrm{s}$ and $E_{n t k p}=374 \mathrm{~V} / \mathrm{m}$ at $(\nabla T)_{0}=1 \mathrm{~K} / \mu \mathrm{m} ; f$ is the relative magnification factor for the maximum flow velocity and it is determined by the numerical value of $u^{\max }$ at $(\nabla T)_{0}=1 \mathrm{~K} / \mu \mathrm{m}$. Note that although analytical expression for the NTKP velocity is described as $u_{n t k p}^{\text {slip }}=2 u_{n t k p}$ (Sugioka 2014b), $u_{n t k p}$ provides the maximum peak value observed in numerical calculations because of the boundary condition that $\boldsymbol{u}=0$.

\section{Results}

\subsection{NTKP vortex flows in Mode 1}

Figure 2 shows the NTKP vortex flows in Mode 1 . Here, $(\nabla T)_{0}=1 \mathrm{~K} / \mu \mathrm{m}, f^{\prime}$ is the relative magnification factor for the flow vector, $T_{0}=W^{2} / D_{0}(=2.5 \mathrm{~s})$ is a diffusion time, $a / W=0.1, W=50 \mu \mathrm{m}, D_{0}=10^{-9} \mathrm{~m}^{2} / \mathrm{s}, u_{c}=W / T_{0}(=20 \mu \mathrm{m} / \mathrm{s})$ is the reference flow velocity, $D_{-}=2.03 D_{0}, \alpha_{+}=2.666, \alpha_{-}=0.106, D_{+}=9.31 D_{0}, Q_{-}=0.53 \mathrm{~kJ} / \mathrm{mol}$, $Q_{+}=13.3 \mathrm{~kJ} / \mathrm{mol}, C_{0}=10^{-7} \mathrm{~mol} / 1, \lambda_{D}=1 \mu \mathrm{m}$, and $F_{0}=623.6$. Note that the same parameters are used through this manuscript except $(\nabla T)_{0}$. At $t / T_{0}=2 \times 10^{-5}$ [in Fig. 2(a)], we initially observe a anomalous vortex flow (AVF) around a metal cylinder; then, at $t / T_{0}=1 \times 10^{-4}$ [in Fig. 2(b)], we observe a standard vortex flow (SVF) at $x>0.3$ and the AVF at $x<0.3$, where the flow direction of the AVF is opposite to that of the SVF (at a steady state). Gradually, the AVF fading out until $t / T_{0} \simeq 2 \times 10^{-4}$; the 


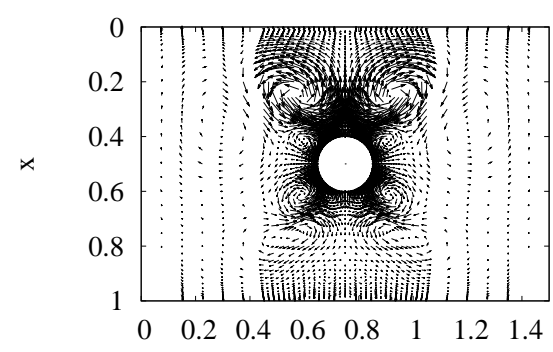

(a) $\mathrm{AVF}$ at $t / T_{0}=2 \times 10^{-5}\left(f^{\prime}=200\right)$

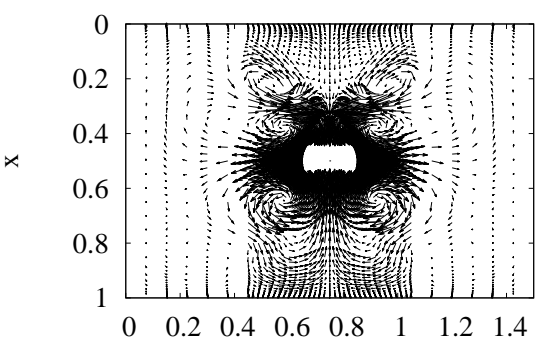

$\mathrm{y}$

(b) AVF and SVF at $t / T_{0}=1 \times 10^{-4}\left(f^{\prime}=\right.$ 30)
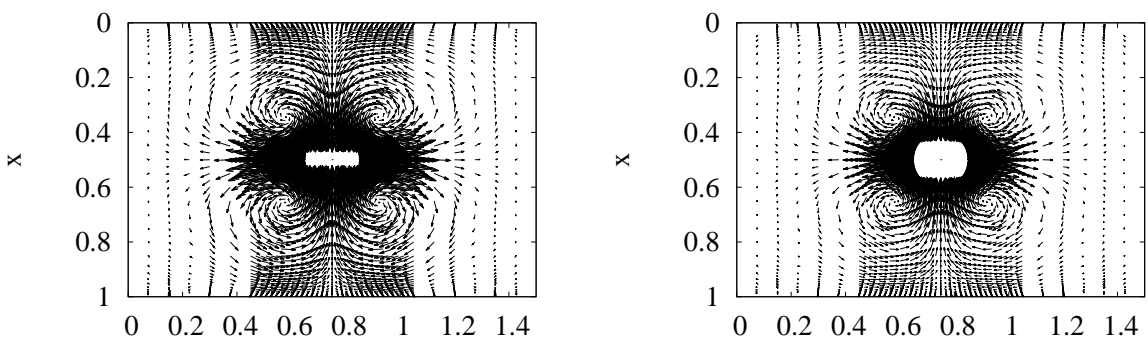

(c) $\mathrm{SVF}$ at $t / T_{0}^{\mathrm{y}}=1.5 \times 10^{-3}\left(f^{\prime}=3\right)$

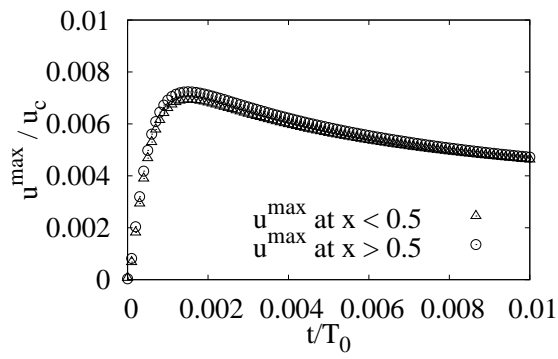

(e) Dependence of $u^{\max }$ on $t$

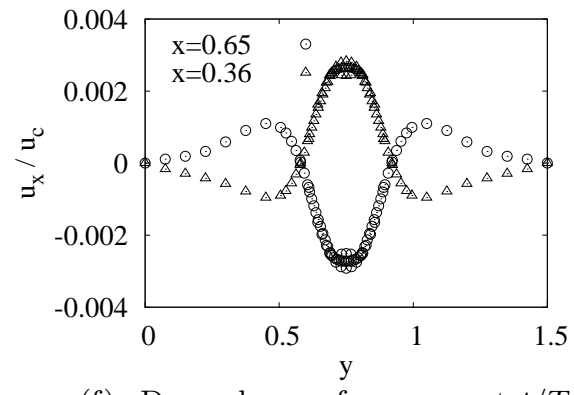

(f) Dependence of $u_{x}$ on $y$ at $t / T_{0}=1 \times$ $10^{-2}$

Figure 2. NTKP vortex flows in Mode 1.

SVF is growing until $t / T_{0} \simeq 1.5 \times 10^{-3}$ [in Fig. 2(c)]; then, the SVF is gradually decaying and it reaches a quasi-steady state at $t / T_{0} \simeq 1 \times 10^{-2}$ [in Fig. 2(d)]. Thus, our previous prediction (Sugioka 2014b) for the NTKP is correct as a whole. Moreover, Fig. 2(e) shows the dependence of $u^{\max }$ on $t$, where $u^{\max }$ is the maximum value of $|\boldsymbol{u}|$. In Fig. 2(e), $u^{\max }$ has the peak value $\left(u^{\text {max,peak }} \simeq 0.007 u_{c}=0.14 \mu \mathrm{m} / \mathrm{s}\right)$ at $t / T_{0}=1.5 \times 10^{-3}$. Further, the flow velocity of the SVF in a steady state is almost symmetrical to the plane of $x=0.5$, as shown in Fig. 2(f). 


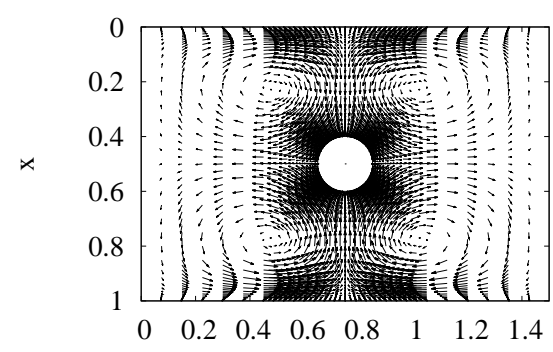

(a) $\mathrm{SVF}$ at $t / T_{0}=2 \times 10^{-5}\left(f^{\prime}=8\right)$

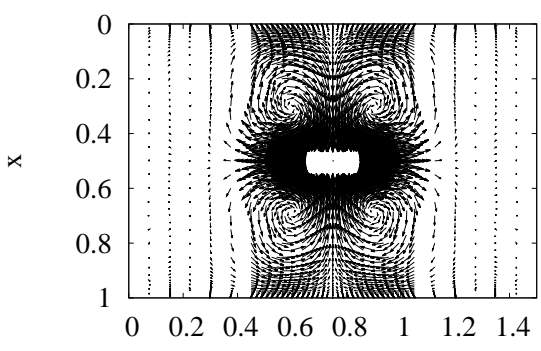

(b) SVF at $t / T_{0}^{\mathrm{y}}=2 \times 10^{-4}\left(f^{\prime}=1\right)$

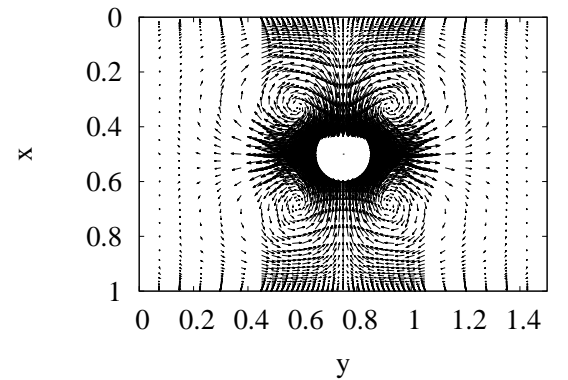

(c) SVF at $t / T_{0}=2 \times 10^{-3} t\left(f^{\prime}=3\right)$

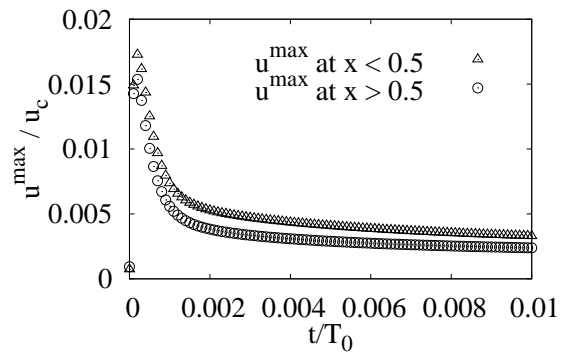

(d) Dependence of $u^{\max }$ on $t$

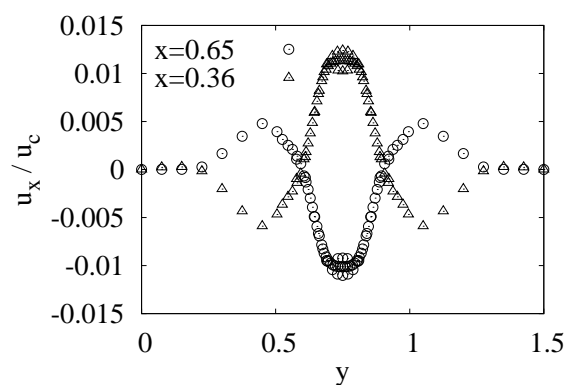

(e) Dependence of $u_{x}$ on $y$ at $t / T_{0}=2 \times$ $10^{-4}$

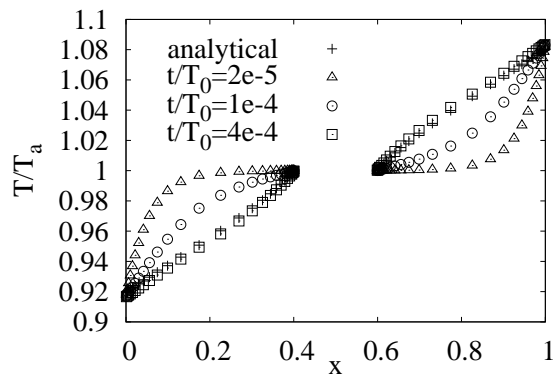

(f) Dependence of $\tilde{T}$ on $x$ at $y=0.75$

Figure 3. NTKP vortex flow in Mode 3.

\subsection{NTKP vortex flows in Mode 3}

Figure 3 shows the NTKP vortex flows in Mode 3. At $t / T_{0}=2 \times 10^{-5}$ [in Fig. 3(a)], different from the result of Mode 1, we initially observe a moderately-strong SVF around a metal cylinder; then, the velocity of the SVF is growing rapidly until $t / T_{0}<2 \times 10^{-4}$ [in Fig. 3(b)]; then, it is decaying with a time constant $\tau^{\text {decay, mode } 3} \simeq 0.002$ and reaches a quasi-steady state at $t / T_{0}=0.002$ [in Fig. 3(c)]. Further, Fig. 3(d) shows the dependence of $u^{\text {max }}$ on $t$. In Fig. 3(d), $u^{\max }$ has the peak values $\left(u^{\text {max }, \text { peak }} \simeq 0.019 u_{c}=0.38 \mu \mathrm{m} / \mathrm{s}\right)$ at $t / T_{0}=2.0 \times 10^{-4}$. Further, from Fig. 3(e), we find that the velocity of the SVF in a peak state is approximately symmetrical to the plane of $x=0.5$ similar to Mode 1 . 
In addition, since $u^{\max }$ of Mode 3 at $x>0.002$ is almost the same as that of Mode 1 , Modes 1 and 3 show a similar characteristic as a whole except the transient period of the temperature at $t / T_{0}<10^{-4}$. Figure $3(\mathrm{f})$ shows the dependence of temperature on $x$ at $y=0.75$ at $t / T_{0}=2.0 \times 10^{-5}, 1.0 \times 10^{-4}$, and $4.0 \times 10^{-4}$. In Fig. 3(f), the numerical results of the temperature distribution at $t / T_{0} \geqslant 0.4 \times 10^{-3}$ is in good agreement with the analytical results. However, the temperature gradient at $t / T_{0}=2.0 \times 10^{-5}$ is very steep; thus, it leads a large thermophoretic velocity at the edges.

\subsection{NTKP vortex flows in Mode 6}

Figure 4 shows the NTKP vortex flows in Mode 4. Surprisingly, different from the result in Mode 3, we just observe a very weak SVF at $t / T_{0}=2 \times 10^{-5}$ in Fig. 4(a); then, observe a 8-pole vortex flow at $t / T_{0}=2 \times 10^{-4}$ and $2 \times 10^{-3}$ in Figs. 4(b) and 4(c). Figure 4(d) shows the dependence of $u^{\max }$ on t. In Fig. 4(d), the response time of Mode 6 is $\sim 0.001 T_{0}$ (i.e., $\tau_{\text {Mode } 6} \simeq 0.001 T_{0}$ ) and $u_{\max }$ at $x<0.5$ is slightly larger than that at $x>0.5$, although $u_{x}$ is almost symmetrical to the plain of $x=0.5$ in Fig. 4(e). Further, in Fig. 4(e), we observe the complex change of the flow direction corresponding to the 8-pole vortex flow. Figure 4(f) shows the dependence of $\phi$ on $x$ for Modes 3 and 6 . From Fig. 4(f), we can recognize that the blocking boundary condition forms much larger potential difference than the passing boundary condition. Here, owing to the blocking boundary condition, the electric field in the $x$ direction due to the Seebeck effect not only suppresses the nonlinear thermokinetic phenomena but also induces the different-type NTKP vortex, which will be discussed later.

\subsection{NTKP characteristics}

Figures. 5(a) and 5(b) show the dependence of $u_{\max }$ on $(\nabla T)_{0}$ for the passing and blocking boundary conditions, respectively. In Fig. 5(a), solid lines denote the analytical results described by Eq. (2.21) with $f=0.19$; i.e., we find that $u_{\max } \propto(\nabla T)_{0}^{2}$ for the passing boundary condition. Further, since the results of a steady state for Modes 1 and 2 agree with each other, we can recognize that the difference of the initial stage does not affect the steady state under the passing boundary condition. In Fig. 5(b), solid and broken lines denote the theoretical curves described by Eq. (2.21) with $f=0.12$ and 0.18 for Modes 4 and 5 , respectively in the region $x>0.5$; i.e., we also find that $u_{\max } \propto(\nabla T)_{0}^{2}$ under the blocking boundary condition. Further, from Figs. 5(a) and 5(b), we find that the difference of the initial behavior affects the flow velocities in Modes 4 and 5 .

Figure 5(c) shows the NTKP at the peak and the broken line denotes the analytical result described by Eq. (2.21) with $f=0.76$. Here, since we theoretically expect $f=1$ for an unbounded problem (Sugioka 2014b), the values of $f(=0.76)$ are acceptable as the first attempt. Figure 5(d) shows the NTKP of Mode 3 and the solid and broken lines denote the analytical results described by Eq. (2.21) with $f=0.096$ and 0.62 , respectively. i.e., we also find that approximately $u^{\max } \propto(\nabla T)_{0}^{2}$ for Mode 3. However, in detail, the numerical results of Mode 3 are slightly different from the relation $\left[u^{\max } \propto(\nabla T)_{0}^{2}\right]$ because of the consideration of the temperature dependences of the parameters.

\subsection{Two kinds of NTKP relevant to the passing and blocking boundary conditions}

Figures 6(a) and 6(b) show the dependence of $\rho$ on $x$ at $y=0.75$ for the passing and blocking boundary conditions, respectively. From Fig. 6, we find that the sign of the charge around the metal cylinder for the blocking boundary condition is the opposite to that for the passing boundary condition. In other words, Fig. 6 clearly shows that there exist two kinds of NTKP corresponding to the passing and blocking boundary 


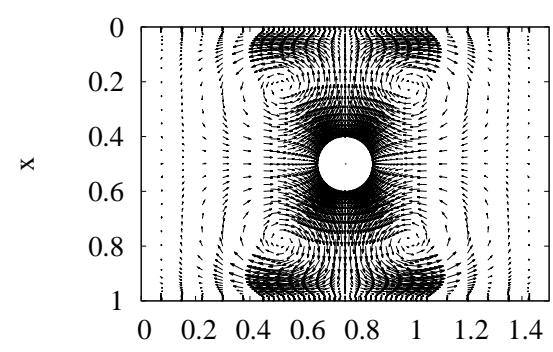

(a) SVF at $t / T_{0}=2 \times 10^{-5}\left(f^{\prime}=400\right)$

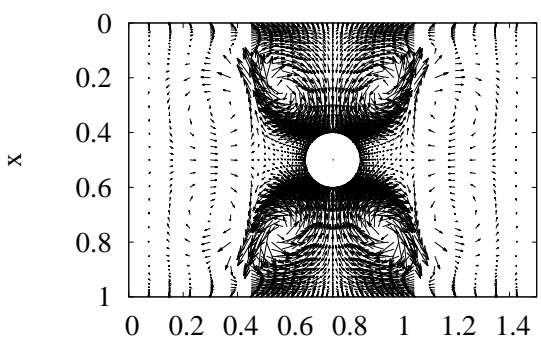

$\mathrm{y}$

(b) 8-pole vortex flow at $t / T_{0}=2 \times 10^{-4}$ $\left(f^{\prime}=15\right)$

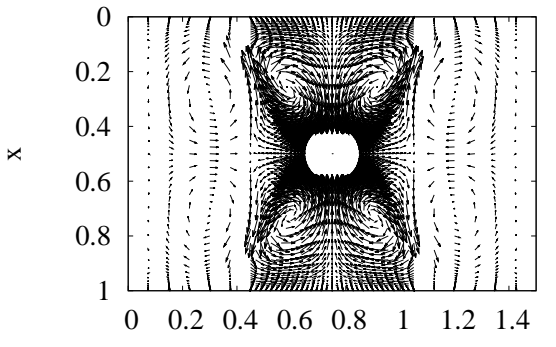

$\mathrm{y}$

(c) 8-pole vortex flow at $t / T_{0}=2 \times 10^{-3}$ $\left(f^{\prime}=3\right)$

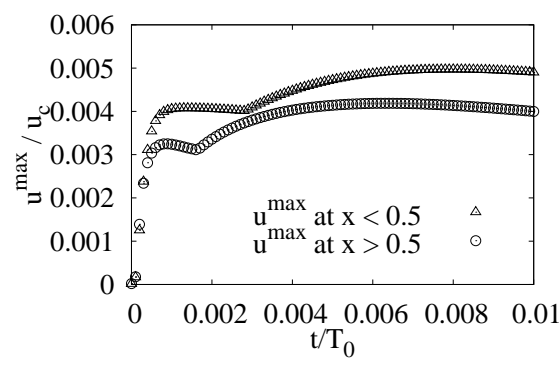

(d) Dependence of $u^{\max }$ on $t$
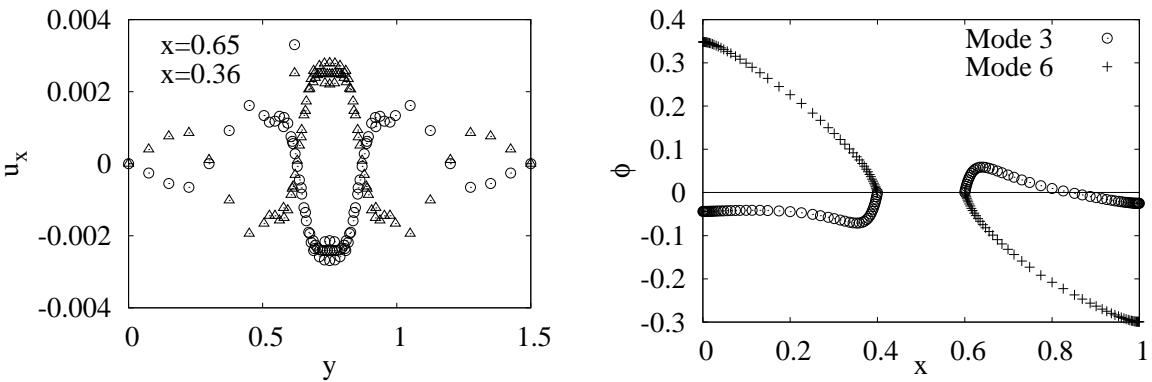

(e) Dependence of $u_{x}$ on $y$ at $t / T_{0}=0.002$ (f) Dependence of $\phi$ on $x$ at $y=0.75$ at $t / T_{0}=0.002$

Figure 4. NTKP vortex flow in Mode 6.

conditions; i.e., Seebeck effect (SE) type NTKP and image effect (IE) type NTKP. Here, the image (charge) effect is a kind of induced charge phenomena of metal and IE-NTKP is approximately explained by the primitive model in Fig. 1. Further, SE-NTKP is the NTKP that is generated by the electric field in the $x$ direction due to the Seebeck effect $\left(\boldsymbol{E}^{\text {Seebeck }}\right)$; thus, because of the polarization of the metal cylinder, the sign of the net ion charge at $x \simeq 0.4$ and 0.6 becomes positive and negative, as shown in Fig. 6(b). Note that in IE-NTKP, the charge on the metal is induced through the image effect by the 


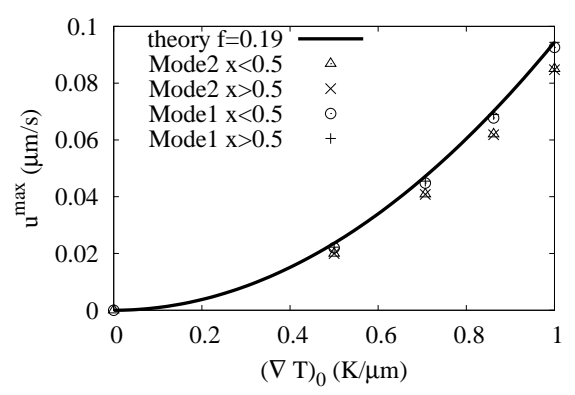

(a) NTKP (passing B.C., $t / T_{0}=0.02$ )

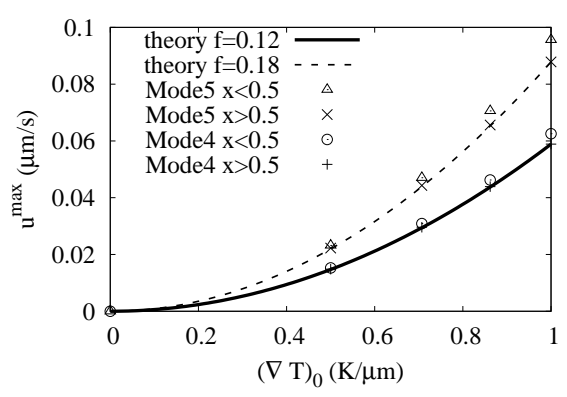

(b) NTKP (blocking B.C., $t / T_{0}=0.02$ )

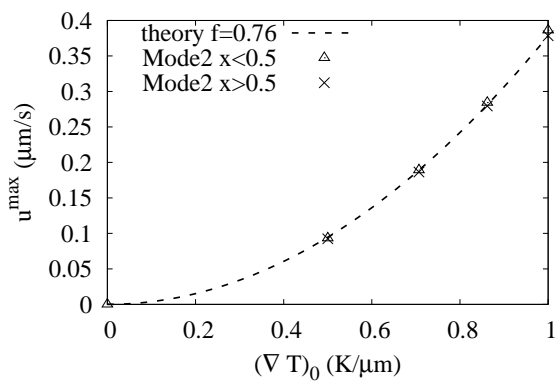

(c) NTKP of Mode 2 at the peak time

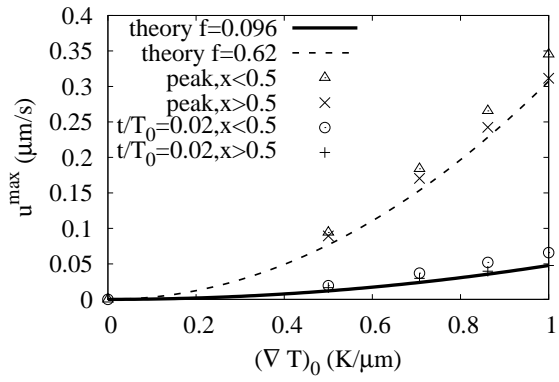

(d) NTKP of Mode 3 at the peak and steady states

Figure 5. Nonlinear dependence of $u_{\max }$ on $(\nabla T)_{0}$.

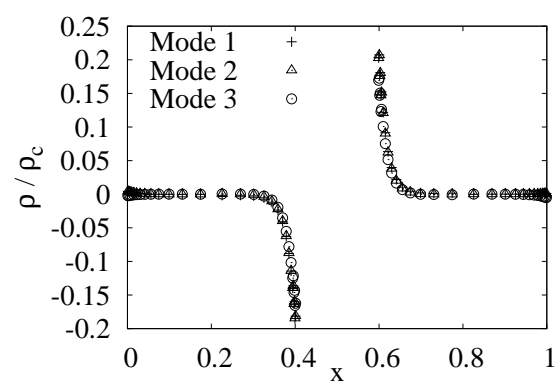

(a) $\rho$ for the passing B.C.

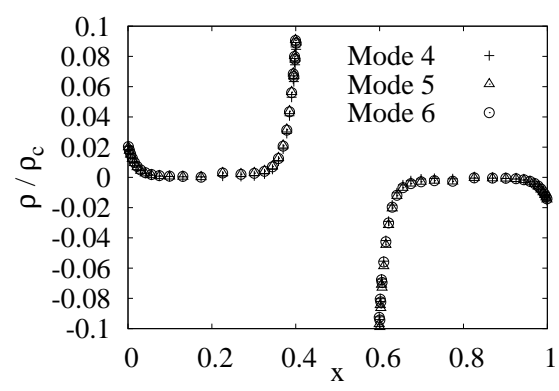

(b) $\rho$ for the blocking B.C.

Figure 6 . Dependence of $\rho_{n}$ on $x$ at $y=0.75$ at $t / T_{0}=0.01$. (a) and (b) show the different origin of the NTKP due to the boundary conditions.

existence of the charge around the metal cylinder, whereas it is induced by the electric field due to the charge near the wall in SE-NTKP.

Figure 7 shows the outside and inside electric fields in Mode 3 and 6. In Figs. 7(b) and $7(\mathrm{~d})$, the direction of the inside electric field in Mode 3 (i.e., IE-NTKP) is opposite to that in Mode 6 (i.e., SE-NTKP). On the one hand, in IE-NTKP (Mode 3), the charge 


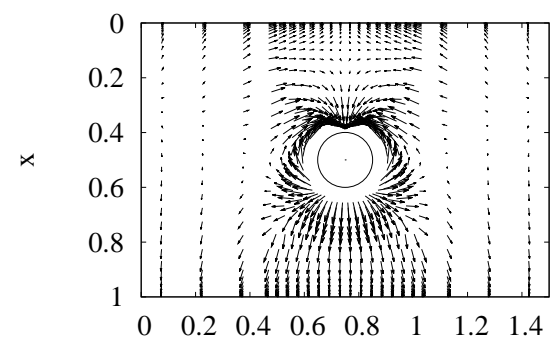

$\mathrm{y}$

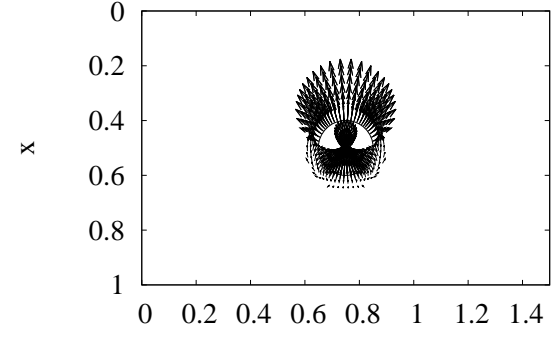

$\mathrm{y}$

(a) Outside electric field in Mode 3 at (b) Inside electric field in Mode 3 at $t / T_{0}=$ $t / T_{0}=0.01 .\left(|\boldsymbol{r}|>1.5 a, f^{\prime \prime}=10\right)$

$0.01\left(|\boldsymbol{r}|<1.5 a, f^{\prime \prime}=2\right)$
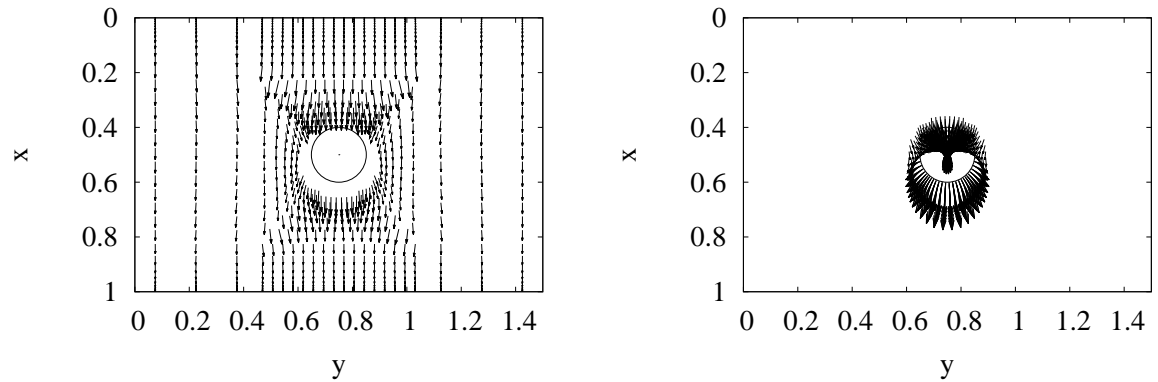

(c) Outside electric field in Mode 6 at (d) Inside electric field in Mode 6 at $t / T_{0}=$ $t / T_{0}=0.002\left(|\boldsymbol{r}|>1.5 a, f^{\prime \prime}=2\right)$ $0.002\left(|\boldsymbol{r}|<1.5 a, f^{\prime \prime}=2\right)$

Figure 7. Outside and inside electric fields in Modes 3 and 6.

around the metal cylinder dominates the phenomena. Thus, the electric field in $|\boldsymbol{r}|>1.5 a$ is much smaller than that in $|\boldsymbol{r}|<1.5 a$ in Figs. 7(a) and 7(b). Further, by considering a positive and negative charge at $x \simeq 0.6$ and 0.4 , we can understand that the direction of the electric fields is reasonable; e.g., the circular electric fields from $x \simeq 0.6$ to $x \simeq 0.4$ is explained in Figs. 7(a) and 7(b). On the other hand, in SE-NTKP (Mode 6), the charge near the wall dominates the phenomena. Thus, the electric field in $|\boldsymbol{r}|>1.5 \mathrm{a}$ is similar to that in $|\boldsymbol{r}|<1.5 a$ in Figs. 7(c) and 7(d). Further, the direction of the outside and inside electric are simply explained by considering the existence of positive and negative charges at $x \simeq 0$ and 1 .

\subsection{Memory and nonsteady effects}

Figure 8 shows the time dependence of $u^{\max }$. In Fig. 8(a), we find a large peak of $u^{\text {max,peak }} \simeq 0.02$ due to the nonsteady effect of Mode 2 . Further, we find that the nonsteady temperature effect disappears approximately at $t / T_{0}>0.002$ and $u^{\text {max }}$ becomes almost the same between Modes 1 and 2. Further, because of the effect of the temperature dependence of $\mu$ and $D_{ \pm}, u^{\text {max } \text {,peak }}$ in Mode 3 becomes 11 to $17 \%$ smaller than that in Mode 2, as shown in Fig. 8(b), although the time dependent behaviors are almost the same qualitatively. Further, in Fig. 8(c), we find the significant memory effect between Modes 4 and 5; i.e., the difference of $u^{\max }$ during the transient state affects the flow velocity of the steady state under the blocking boundary condition [in Fig. 8(c)], whereas the difference of the transient state does not affect the flow velocity of the steady 


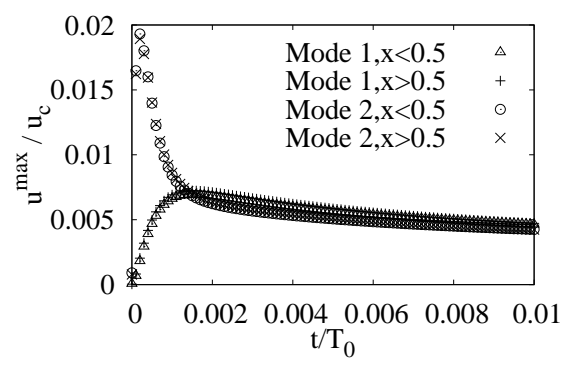

(a) Modes 1 and 2 (passing b.c.)

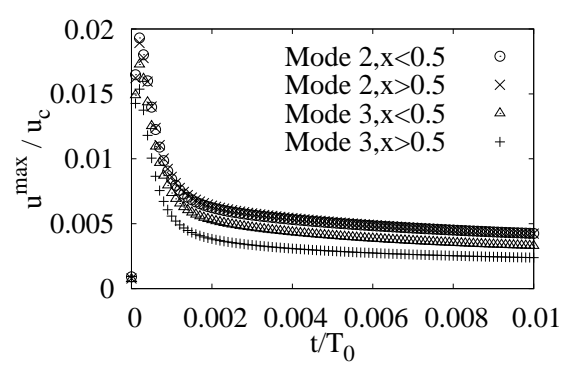

(b) Modes 2 and 3 (passing b.c.)

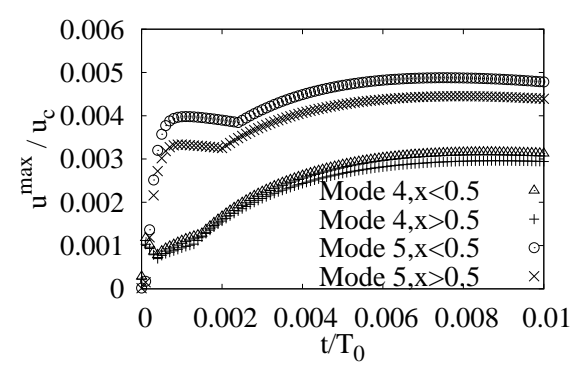

(c) Modes 4 and 5 (blocking b.c)

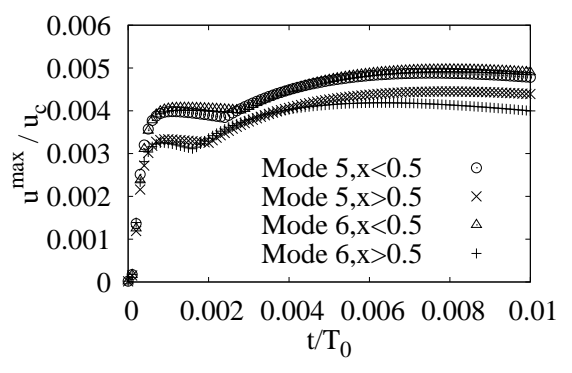

(d) Modes 5 and 6 (blocking b.c.)

FiguRE 8 . Time dependence of $u^{\max }$ (memory and nonsteady effects).

state under the passing ion condition [in Fig. 8(a)]. In addition, in Fig. 8(d), the time dependent behaviors are almost the same between Modes 5 and 6 ; i.e., the effect using the temperature dependence of the parameter is rather small for the blocking boundary condition.

Figures $9(\mathrm{a})$ and $9(\mathrm{~b})$ show the time evolution the maximum value of $\rho$ (i.e., $\rho^{\max }$ ) at $x>0.5$. We find that the memory effect on $\rho^{\max }$ exists for the blocking boundary condition, whereas it does not exit for the passing boundary condition. Further, Figs. 9(c) and $9(\mathrm{~d})$ show the dependence of $\rho$ on $x$ at $y=0.6$ at $t / T_{0}=0.01$. In Fig. $9(\mathrm{~d})$, we find that a significant difference of $\rho$ exists between Modes 4 and 5 , whereas the difference of $\rho$ is rather small between Modes 1 and 2 in Fig. 9(c). Note that the dependence of $\rho$ on $x$ at $y=0.75$ (i.e., at the mid position) for Mode 5 is the same as that for mode 4 at $t / T=0.01$, as shown in Fig. 6(b); thus, we consider that the hysteresis of $\rho$ [in Figs. 9(b) and $9(\mathrm{~d})$ ] causes the memory effect of Modes 4 to 6 .

\subsection{Time evolution of the ion distributions in Modes 3 and 6}

Figures 10 shows the dependence of $C_{+}, C_{-}, \rho$, and $\phi$ on $x$ at $y=0.75$ at $t / T_{0}=0.0002$, 0.002 , and 0.01 in Mode 3. Since the thermal mobility factor of $\mathrm{H}^{+}\left(2 \alpha_{+} D_{+}=49.6 D_{0}\right)$ is much larger than that of $\mathrm{Cl}^{-}\left(2 \alpha_{-} D_{-}=0.431 D_{0}\right)$ (Majee \& Würger 2012; Agar et al. 1989), the positive ion $\left(\mathrm{H}^{+}\right)$moves from a hotter region to a colder region with a much faster velocity than the negative ion $\left(\mathrm{Cl}^{-}\right)$. Thus, in Figs. 10(a) and 10(b), $C_{+}$becomes much larger than $C_{-}$at $x \simeq 0.6$ whereas $C_{-}$becomes much larger than $C_{+}$at $x \simeq 0.4$. Consequently, in Fig. 10(c), we can observe a positive charge region at the hotter side of 


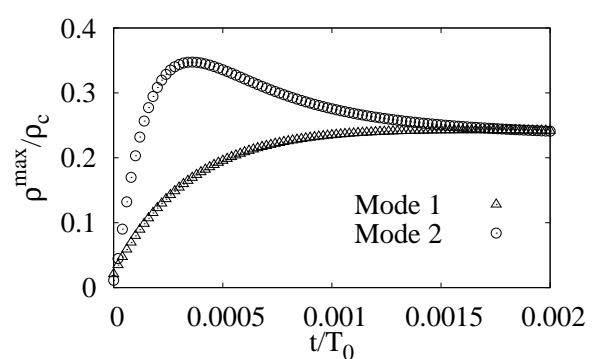

(a) $\rho$ for the passing B.C. $(x>0.5)$

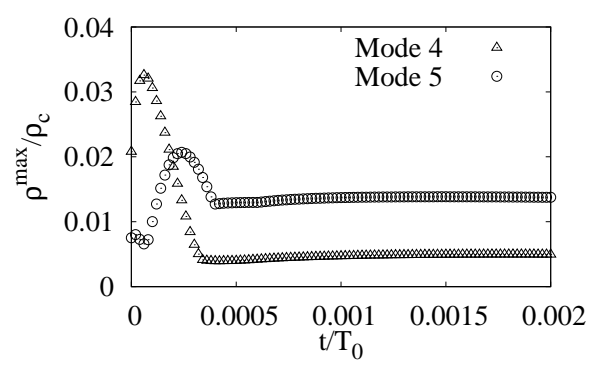

(b) $\rho$ for the blocking B.C. $(x>0.5)$

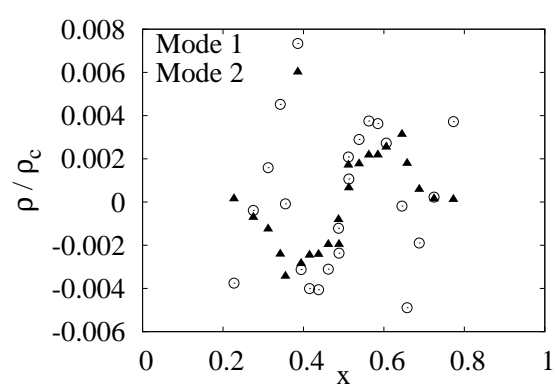

(c) $\rho$ for the passing B.C. $(y=0.6)$

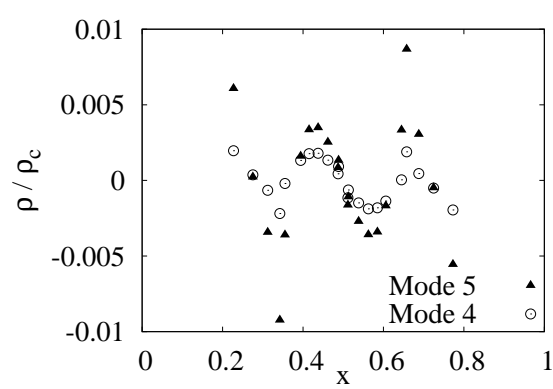

(d) $\rho$ for the blocking B.C. $(y=0.6)$

Figure 9. The time evolution of $\rho^{\max }$ at $x>0.5$ and the dependence of $\rho$ on $x$ at $t / T_{0}=0.01$.

the metal cylinder, whereas we can observe a negative charge region at the colder side. In detail, the time evolution of $C_{-}$around the metal cylinder [in Fig. 10(b)] is complex and it should be explained by the image charge effect of the positive ion $\left(\mathrm{H}^{+}\right)$. Further, we find that the arrival of the fast ion in the initial stage (at $t / T_{0}=0.0002$ ) induces a large transient charge density around metal through the image charge effect and a large electric field in the $-x$ direction, whereas the arrival of the slow ion in the final stage (at $t / T_{0}=0.01$ ) suppresses the charge and the electric field around the metal. This is the reason why $u^{\max }$ in Modes 2 and 3 is large in the initial stage and suppressed in the final stage [in Fig. 8(b)]. Note that in Mode 3, the concentrations of ions at the edges are almost the same as the bulk concentration [in Figs. 10(a) and 10(b)] and the charges at edges are almost zero [in Fig. 10(c)].

Figures 11 shows the dependences of $C_{+}, C_{-}, \rho$, and $\phi$ on $x$ at $y=0.75$ at $t / T_{0}=$ $0.0002,0.002$, and 0.01 in Mode 6. Since ions can not go through the edges in Mode 6 , the concentration of ions at the colder (hotter) edge is larger (smaller) than the bulk concentration [in Figs. 11(a) and 11(b)] and the charge at the colder (hotter) edge becomes positive (negative) in Mode 6 [in Fig. 11(c)]. As a result, the potential difference between the edges becomes large in Mode 6 [in Fig. 11(d)]. Thus, the large electric field in the $x$ direction is generated through the Seebeck effect and it drives ICEO phenomena around the metal. 


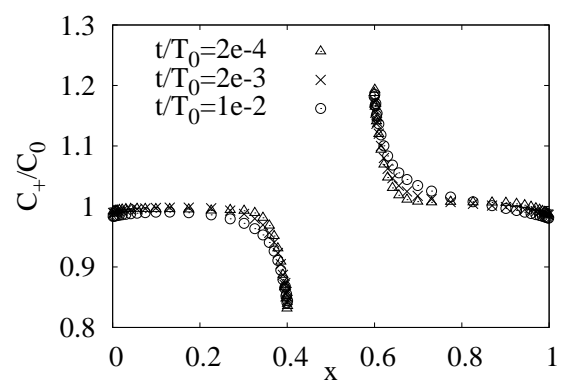

(a) Dependence of $C_{+}$on $x$ at $y=0.75$

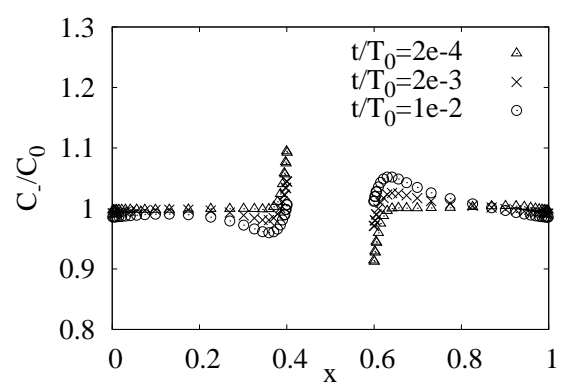

(b) Dependence of $C_{-}$on $x$ at $y=0.75$

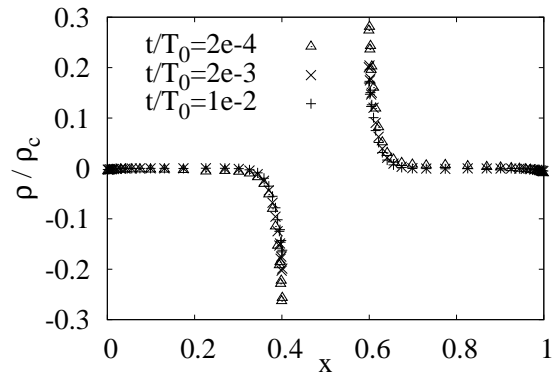

(c) Dependence of $\rho_{e}$ on $x$ vat $y=0.75$

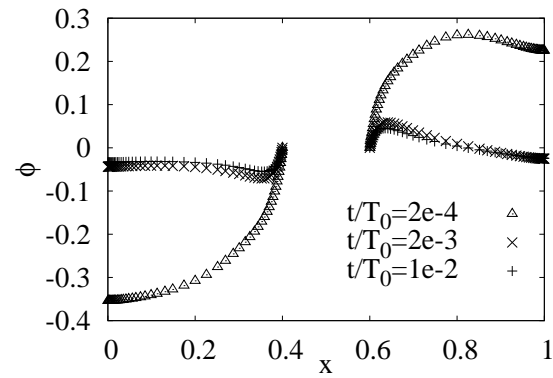

(d) Dependence of $\phi$ on $x$ at $y=0.75$

Figure 10. Time evolution of the ion distributions in Mode 3 (IE-NTKP).

\section{Discussion}

4.1. The gradient of ion concentration for the blocking boundary condition at the wall

If we can neglect the effect of the charge of ions, we can estimate the gradient of ion concentrations due to thermokinetic phenomena for the blocking boundary condition by using Eqs. (2.8) and (2.13) as follows:

$$
\begin{aligned}
& \nabla_{n} c_{+}=-2 \frac{\alpha_{+}}{\tilde{T}} c_{+} \frac{\nabla_{n} \tilde{T}}{\tilde{T}} \simeq-2 \alpha_{+}\left(\nabla_{n} \tilde{T}\right)_{0} \\
& \nabla_{n} c_{-}=-2 \frac{\alpha_{-}}{\tilde{T}} c_{-} \frac{\nabla_{n} \tilde{T}}{\tilde{T}} \simeq-2 \alpha_{-}\left(\nabla_{n} \tilde{T}\right)_{0}
\end{aligned}
$$

where $\alpha_{+} \simeq 2.7\left(\right.$ for $\left.\mathrm{H}^{+}\right), \alpha_{-} \simeq 0.1\left(\right.$ for $\left.\mathrm{Cl}^{-}\right), \nabla_{n} \tilde{T} \simeq\left(\nabla_{n} \tilde{T}\right)_{0}[=(1 \mathrm{~K} / 1 \mu \mathrm{m}) \times$ $(50 \mu \mathrm{m} / 300 \mathrm{~K})=0.167], \tilde{T} \simeq 1$, and $c_{ \pm} \simeq 1$. That is, $\nabla_{n} c_{+} \gg \nabla_{n} c_{-}$in the first stage and the tendency to become $\nabla_{n} c_{+} \simeq-2 \times 2.7 \times 0.167 \simeq-0.9$ and $\nabla_{n} c_{-}-2 \times 0.1 \times 0.167 \simeq$ -0.03 is a basic driving force of the phenomena. However, since the difference of $\nabla_{n} c_{ \pm}$ produces a net space charge, we need to consider the effect of electric field on the ion distributions. Namely, by using the numerical result $\left(\nabla_{n} \phi\right)_{c} \simeq-0.6$ in Fig. 11(d), we can estimate as follows:

$$
\nabla_{n} c_{+}=-2 \frac{\alpha_{+}}{\tilde{T}} c_{+} \frac{\nabla_{n} \tilde{T}}{\tilde{T}}-c_{ \pm} \frac{\nabla_{n} \phi}{\tilde{T}} \simeq-2 \alpha_{+}\left(\nabla_{n} \tilde{T}\right)_{0}-\left(\nabla_{n} \phi\right)_{c}
$$

Since the numerical value of $\nabla_{n} c_{+}$is approximately $-0.35(\simeq-0.07 / 0.2)$ in Fig. 11(a), the estimation $\left(\nabla_{n} c_{+} \simeq-2 \times 2.7 \times 0.167+0.6 \simeq-0.3\right)$ of Eq. (4.3) is reasonable. Note 


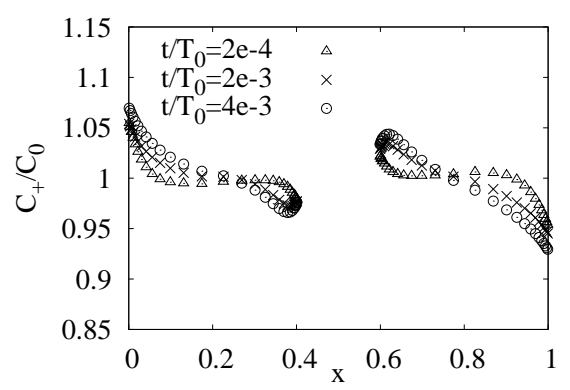

(a) Dependence of $C_{+}$on $x$ at $y=0.75$

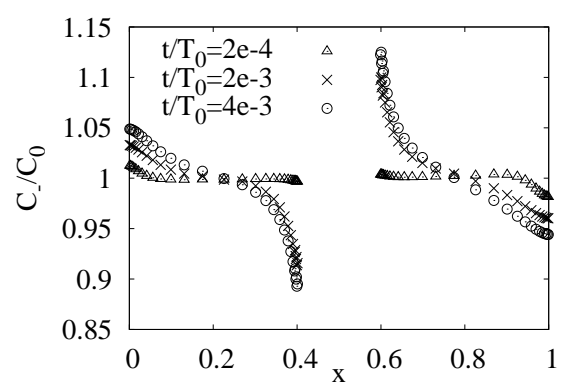

(b) Dependence of $C_{-}$on $x$ at $y=0.75$

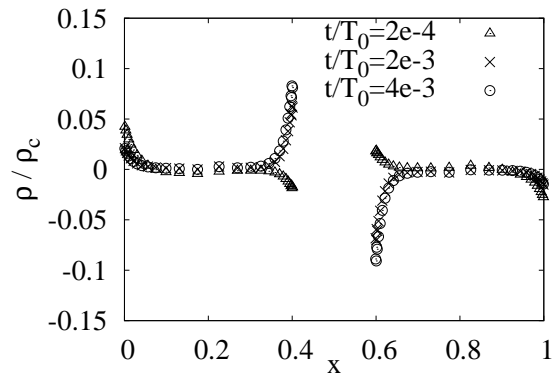

(c) Dependence of $\rho_{e}$ on $x$ vat $y=0.75$

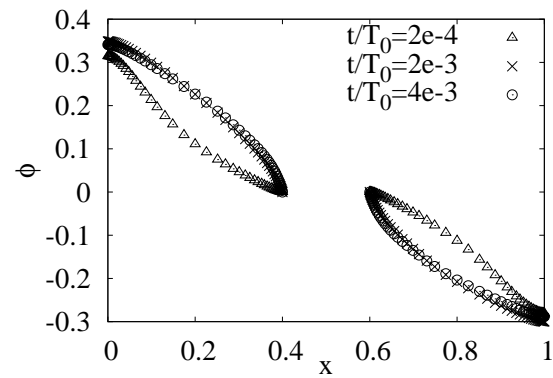

(d) Dependence of $\phi$ on $x$ at $y=0.75$

Figure 11. Time evolution of the ion distributions in Mode 6 (SE-NTKP).

that the generation of the electric field due to the thermophoresis also affects $\nabla_{n} c_{-}$and it increases the gradient of $c_{-}$. Thus, $\nabla_{n} c_{-}$becomes the value similar to $\nabla_{n} c_{+}$in the bulk region and the existence of charge is limited in the wall region which thickness is approximately $\lambda_{D}$, as shown in Figs. 11(a) to 11(c). Usually, this process is simply explained by the Seebeck effect described by Eq. (2.24), which is derived from Eqs. (2.8) under the zero current condition and the assumption $c_{+} \simeq c_{-}$in the bulk region. Thus, $\nabla_{n} \phi$ can be estimated by Eq. (2.24). For instance, since $S_{e}=374 \mu \mathrm{V} / \mathrm{K}$ for a $\mathrm{HCl}$ solution, the potential difference $\Delta \phi=-374 \times 50 \simeq-18.7 \mathrm{mV}\left(-0.72 \Phi_{c}\right)$ for $W=50 \mu \mathrm{m}$; thus, we obtain $\nabla_{n} \phi \simeq-0.72$ and it approximately agrees with the result of Fig. 11(d) (i.e., $\nabla_{n} \phi \simeq-0.6$ and $\Delta \phi \simeq 0.6$ ), where $\Phi_{c}=k T_{a} / z e=25.9 \mathrm{mV}$.

\subsection{The influence of the electrolyte concentration near the wall}

Since $\lambda_{D} \equiv \sqrt{\varepsilon k T_{a} / 2 z^{2} e^{2} C_{0}}, \lambda_{D}$ decreases as $C_{0}$ increases. As discussed in Sec. 4.1, for the blocking boundary condition, $c_{ \pm}$(and $\nabla_{n} c_{ \pm}$) becomes the same value except the wall region of thickness $\lambda_{D}$ because of the interaction between the positive and negative ions with the screening effect of the electric field. Moreover, in our understanding, $\nabla_{n} c_{+}$and $\nabla_{n} c_{-}$near the wall region are approximated by the maximum gradient of $c_{+}$of Eq. (4.1) and zero, respectively; i.e., $\nabla_{n} c_{+}^{\text {wall }} \simeq-0.9$ and $\nabla_{n} c_{-}^{\text {wall }} \simeq 0$. Thus, we estimate the maximum charge density near the wall as

$$
\rho^{\text {wall }} / \rho_{c} \simeq\left|\nabla_{n} c_{+}^{\text {wall }}\right| \frac{\lambda_{D}}{W} \simeq\left|2 \alpha_{+}\left(\nabla_{n} \tilde{T}\right)_{0}\right| \frac{\lambda_{D}}{W}
$$


where $\left|2 \alpha_{+}\left(\nabla_{n} \tilde{T}\right)_{0}\right| \simeq 0.9, \lambda_{D}=1 \mu \mathrm{m}$, and $W=50 \mu \mathrm{m}$; i.e., $\rho^{\text {wall }} / \rho_{c} \simeq 0.02$. Since the numerical result of $\rho^{\text {wall }} / \rho_{c}$ at $x=0$ for the steady state in Fig. $11(\mathrm{c})$ is also $\simeq 0.02$, our simple argument of Eq. (4.4) explains the thermokinetic phenomena near the wall. to some extent.

Further, the charge per unit area near the wall is written as $\sigma^{\text {wall }}=\rho^{\text {wall }} \lambda_{D}$. Thus, the electric field produced by the charge near the wall in SE-NTKP is described as

$$
E_{s e(1)} \simeq \frac{\rho^{\text {wall }} \lambda_{D}}{\varepsilon} \simeq 2 \alpha_{+}\left(\nabla_{n} \tilde{T}\right)_{0} \frac{\lambda_{D}^{2}}{W \varepsilon} \rho_{c}
$$

Namely, for the current condition, we obtain $E_{s e(1)}=245.7 \mathrm{~V} / \mathrm{m}$ and find that the potential difference across the chamber is $\Delta \Phi=E_{s e(1)} W=12.3 \mathrm{mV}\left(\sim 0.5 \Phi_{c}\right)$. Since it approximately agrees with the numerical result of $\Delta \Phi \simeq 0.6 \Phi_{c}$ in Fig. 11(d), Eq. (4.5) is reasonable. Consequently, according to our argument, we can understand that $E_{s e(1)}$ and $\sigma^{\text {wall }}$ does not change even if $C_{0}$ changes, although $\rho^{\text {wall }}$ is proportional to $\sqrt{C_{0}}$.

\subsection{The gradient of ion concentration around the metal cylinder in IE-NTKP}

From Eq. (2.1), we obtain

$$
\frac{\partial T}{\partial r} \simeq\left(1+\frac{a^{2}}{r^{2}}\right)(\nabla T)_{0} \cos \theta
$$

Thus, we can approximate that $\nabla_{n} \tilde{T} \simeq 2\left(\nabla_{n} \tilde{T}\right)_{0}=0.334$ at $x=0.4$ and 0.6 . Therefore, by using a similar argument in Sec. 4.1, if we can neglect the charge of particles and the image effect of the metal, we can estimate the gradient of ions around the metal as follows:

$$
\begin{aligned}
\nabla_{n} c_{+}^{\text {metal }} & \simeq-4 \alpha_{+}\left(\nabla_{n} \tilde{T}\right)_{0} \\
\nabla_{n} c_{-}^{\text {metal }} & \simeq-4 \alpha_{-}\left(\nabla_{n} \tilde{T}\right)_{0}
\end{aligned}
$$

That is, the tendency to become $\nabla_{n} c_{+}^{\text {metal }} \simeq-1.8$ and $\nabla_{n} c_{-}^{\text {metal }} \simeq-0.06$ is a basic driving force of the phenomena around the metal cylinder. Different from the argument of Sec. 4.1, we can neglect the second term of Eq. (4.3) because of the existence of the image charge of the metal cylinder, as a first step. Further, since $\nabla_{n} c_{+} \simeq-0.2 / 0.1 \simeq-2$ at $x \simeq 0.4$ and 0.6 in Fig. 10(a), the estimation of Eq. (4.7) is reasonable. Further, similar to the discussion in Sec. 4.1 and $4.2, c_{ \pm}$(and $\nabla_{n} c_{ \pm}$) becomes the same value except the surrounding region of the metal cylinder of thickness $\lambda_{D}$ because of the interaction between the positive and negative ions with the screening effect of the electric field. However, because of the existence of the image charge of the metal cylinder, the maximum charge density near the metal cylinder becomes two times of that near the unpolarizable wall;

$$
\rho_{i e . m(1)} / \rho_{c} \simeq 8 \alpha_{+}\left(\nabla_{n} \tilde{T}\right)_{0} \frac{\lambda_{D}}{W}
$$

In fact, different from $c_{-}$at $x \simeq 0.6$ in Fig. $11(\mathrm{~b}), c_{-}$at $x \simeq 0.6$ in Fig. 10 (b) decreases as the position approaches to the surface of the metal because of the image effect and thus the charge increases rapidly near the metal. In addition, because of the polarization effect of the metal due to the existence of the excess counter ions at the other side, the charge density of Eq. (4.9) becomes two times again; i.e.,

$$
\rho_{i e . m(2)} / \rho_{c} \simeq 16 \alpha_{+}\left(\nabla_{n} \tilde{T}\right)_{0} \frac{\lambda_{D}}{W} .
$$


Since $\rho_{i e . m(2)} / \rho_{c} \simeq 0.014$, the numerical result of $\rho / \rho_{c} \sim 0.2$ at $x \simeq 0.6$ in Fig. 10 (c) is approximately explained by our simple argument. Further, since $\rho_{i e . m(2)} \lambda_{D}$ represents the charge per unit area at the hotter side of the metal, we can estimate the zeta potential at $x \simeq 0.6$ in IE-NTKP as

$$
\zeta_{i e}^{\prime} \simeq \frac{\lambda_{D}^{2}}{\varepsilon} \rho_{i e . m(2)} \simeq 16 \alpha_{+}\left(\nabla_{n} \tilde{T}\right)_{0} \frac{\lambda_{D}^{3} \rho_{c}}{\varepsilon W} .
$$

Here, $\zeta_{i e}^{\prime}$ corresponds to the peak value of $\phi(\simeq 0.07)$ at $x \simeq 0.6$ in Fig. 10(d); thus, $\zeta_{i e}^{\prime} \simeq 2.0 \mathrm{mV}\left(0.076 \Phi_{c}\right)$ in Eq. (4.11) explains it to some extent.

\subsection{Theory on IE-NTKP}

Based on the Smoluchowski formula, we can describe the IE-NTKP flow velocity as

$$
u_{i e}^{s l i p}=\frac{\varepsilon \zeta_{i e}^{\prime \prime}}{\mu} E_{i e},
$$

where $E_{i e}$ denotes an apparent external electric field in IE-NTKP, which acts on the charge in the electric double layer around the metal cylinder, and $\zeta_{i e}^{\prime \prime}$ is a zeta potential around the metal at the $\theta=45^{\circ}$ in IE-NTKP. Since $\zeta \propto \cos \theta$ at around the metal cylinder, we can approximate

$$
\zeta_{i e}^{\prime \prime} \simeq \frac{\zeta_{i e}^{\prime}}{\sqrt{2}} \simeq 8 \sqrt{2} \alpha_{+}\left(\nabla_{n} \tilde{T}\right)_{0} \frac{\lambda_{D}^{3} \rho_{c}}{\varepsilon W}
$$

Further, the electric field in Fig. 7(a) shows that $E_{i e}$ in IE-NTKP should be described as

$$
E_{i e} \simeq-\frac{2 \zeta_{i e}^{\prime}}{\pi\left(a+\lambda_{D}\right)} \simeq-32 \alpha_{+}\left(\nabla_{n} \tilde{T}\right)_{0} \frac{\lambda_{D}^{3} \rho_{c}}{\varepsilon W}
$$

By substituting Eqs. (4.13) and (4.14) into Eq. (4.12), we obtain

$$
u_{i e}^{\text {slip }} \simeq-\frac{\sqrt{2} \varepsilon \zeta^{\prime 2}}{\pi\left(a+\lambda_{D}\right) \mu}
$$

Thus, the numerically observable maximum velocity is provided as

$$
u_{i e} \simeq \frac{1}{2} u_{i e}^{\text {slip }} \simeq-\frac{\varepsilon\left[16 \alpha_{+}\left(\nabla_{n} \tilde{T}\right)_{0} \frac{\lambda_{D}^{3} \rho_{c}}{\varepsilon W}\right]^{2}}{\sqrt{2} \pi\left(a+\lambda_{D}\right) \mu}
$$

In Eqs. (4.15) and (4.16), a negative value shows the flow in the $-x$ direction near $x \simeq 0.6$. From Eqs. (4.13), (4.14), and (4.16), we obtain that $\zeta_{i e}^{\prime \prime} \simeq 0.054 \Phi_{c} \simeq 1.4 \mathrm{mV}, E_{i e} \simeq-209$ $\mathrm{V} / \mathrm{m}$, and $u_{i e} \simeq-0.051 u_{c} \simeq-0.10 \mu \mathrm{m} / \mathrm{s}$, under the current conditions. Numerical calculation shows that $u^{\max } \simeq 0.19 u_{n t k p} \simeq 0.094 \mu \mathrm{m} / \mathrm{s}$ for IE-NTKP in Fig. 5(a). Thus, Eq. (4.16) explains the flow velocity of IE-NTKP reasonably. Consequently, our new theory on IE-NTKP is justified to some extent.

\subsection{Theory on SE-NTKP}

By the Smoluchowski formula, the SE-NTKP flow velocity is described as

$$
u_{s e}^{s l i p}=\frac{\varepsilon \zeta_{s e}^{\prime \prime}}{\mu} E_{s e},
$$

where $E_{\text {se }}$ denotes an apparent external electric field in SE-NTKP, which acts on the charge in the electric double layer around the metal cylinder, and $\zeta_{s e}^{\prime \prime}$ is a zeta potential 
around the metal at the $\theta=45^{\circ}(x \simeq 0.576)$ in SE-NTKP. Further, SE-NTKP is mainly driven by the charge near the wall and thus, on the basis of ICEO theory, the zeta potential due to $E^{s e(1)}$ [provided by Eq. (4.5)] at the hotter side of the metal is described as

$$
\zeta_{s e . m(1)}^{\prime} \simeq-2 a E_{s e(1)} .
$$

Furthermore, since $\sigma=\frac{\varepsilon}{\lambda_{D}} \zeta_{s e . m(1)}^{\prime}$, the charge density due to $E_{s e(1)}$ is described as

$$
\rho_{\text {se.m }(1)} \simeq-\frac{2 a E_{s e(1)} \varepsilon}{\lambda_{D}^{2}} .
$$

However, there exists an intrinsic charge density $\rho_{\text {se.m(2) }}$ due to the thermophoretic force at the hotter side of the metal in SE-NTKP. That is, similar to the discussion of Eqs. (4.6) and (4.7), it is described as

$$
\rho_{\text {se.m(2) }} \simeq 8 \alpha_{+}\left(\nabla_{n} \tilde{T}\right)_{0} \rho_{c} \frac{\lambda_{D}}{W} .
$$

Note that there is no counter ions at the other side in SE-NTKP. Thus, $\rho_{\text {se.m(2) }}=$ $0.5 \rho_{\text {ie.m(2) }}$. Therefore, by considering the principle of superposition, we obtain a net charge density at the hotter side of the metal in SE-NTKP as

$$
\rho_{\text {se.m }} \simeq \rho_{\text {se.m(1) }}+\rho_{\text {se.m(2) }} .
$$

Thus, the corresponding zeta potential is

$$
\zeta_{s e}^{\prime} \simeq \frac{\lambda_{D}^{2} \rho_{s e . m}}{\varepsilon}
$$

As discussed before, we can approximate

$$
\zeta_{s e}^{\prime \prime} \simeq \frac{\zeta_{s e}^{\prime}}{\sqrt{2}} \simeq 2 \sqrt{2} \alpha_{+}\left(\nabla_{n} \tilde{T}\right)_{0} \frac{\lambda_{D}^{2} \rho_{c}}{\varepsilon}\left(-\frac{a}{W}+2 \frac{\lambda_{D}}{W}\right) .
$$

In addition, by using Eq. (4.20), the intrinsic zeta potential around the metal due to $\rho_{\text {se.m(2) }}$ is described as

$$
\zeta_{s e(2)}^{\prime} \simeq \frac{\lambda_{D}^{2} \rho_{s e . m(2)}}{\varepsilon} .
$$

Thus, the intrinsic electric field $E_{s e(2)}$ due to $\zeta_{s e . m(2)}^{\prime}$ in SE-NTKP is described as

$$
E_{s e(2)} \simeq-\frac{2 \zeta_{s e(2)}^{\prime}}{\pi\left(a+\lambda_{D}\right)}\left(=\frac{1}{2} E_{i e}\right) .
$$

Since the electric field outside the electric double layer around the metal at $\theta$ is described as $E=2 E_{0} \sin \theta$ using the external average electric field $E_{0}$ in standard ICEO theory (Squires \& Bazant 2004; Sugioka 2011), we obtain

$$
E_{s e} \simeq 2\left(E_{s e(1)}+E_{s e(2)}\right) \sin \frac{\pi}{4}=2 \sqrt{2} \alpha_{+}\left(\nabla_{n} \tilde{T}\right)_{0} \frac{\lambda_{D}^{2} \rho_{c}}{W \varepsilon}\left[1-\frac{8 \lambda_{D}}{\pi\left(a+\lambda_{D}\right)}\right] .
$$

Therefore, by substituting Eqs. (4.23) and (4.26) into Eq. (4.17), we obtain

$$
u_{s e}^{s l i p} \simeq 4 \frac{\varepsilon}{\mu}\left[\alpha_{+}\left(\nabla_{n} \tilde{T}\right)_{0}\right]^{2}\left(\frac{\lambda_{D}^{2} \rho_{c}}{W \varepsilon}\right)^{2} W\left(-\frac{a}{W}+2 \frac{\lambda_{D}}{W}\right)\left[1-\frac{8 \lambda_{D}}{\pi\left(a+\lambda_{D}\right)}\right] .
$$

Further, the observable maximum velocity is provided as

$$
u_{s e} \simeq \frac{1}{2} u_{s e}^{s l i p} .
$$




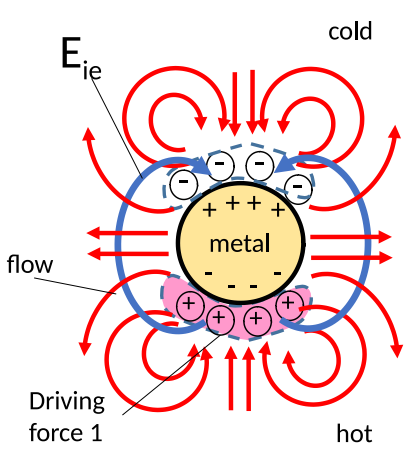

(a) IE-NTKP

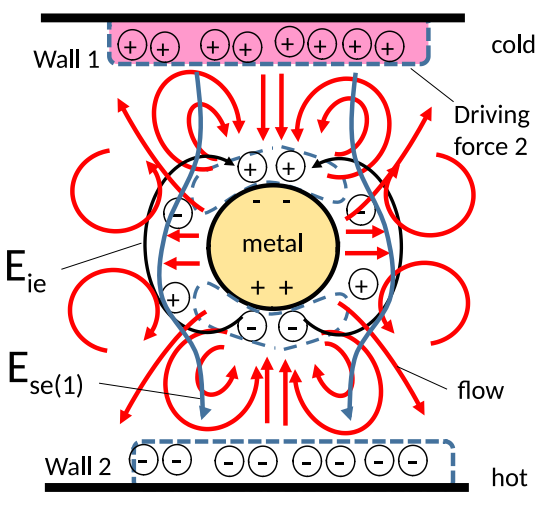

(b) SE-NTKP (8-pole vortex flow)

Figure 12. (Color online) Physical pictures on IE- and SE-NTKP.

Thus, from Eqs. (4.23), (4.26), and (4.28), we obtain $\zeta_{s e}^{\prime \prime} \simeq 0.040 \Phi_{c}=-1.0 \mathrm{mV}, E_{s e} \simeq$ $200 \mathrm{~V} / \mathrm{m}$, and $u_{s e} \simeq-0.0037 u_{c} \simeq-0.074 \mu \mathrm{m} / \mathrm{s}$. Numerical calculation shows that $u^{\max }=f_{4} u_{n t k p} \simeq 0.12 u_{n t k p}$ for Mode 4 and $u^{\max }=f_{5} u_{n t k p} \simeq 0.18 u_{n t k p}$ for Mode 5 for SE-NTKP [in Fig. 5(b)]. That is, the average relative magnification factor $\bar{f}_{e 5}=$ $\left(f_{4}+f_{5}\right) / 2=0.15$. Since $0.15 u_{n t k p} \simeq 0.074 \mu \mathrm{m} / \mathrm{s}$, Eq. (4.28) explains the flow velocity of SE-NTKP reasonably. Consequently, our new theory on SE-NTKP is justified to some extent.

\subsection{Summary of physical pictures on IE- and SE-NTKP}

Figure 12 shows physical pictures on IE- and SE-NTKP. Namely, on the one hand, as shown in Fig. 12(a), IE-NTKP is driven by the excess positive ion that is pushed on the hotter side of the metal by the thermophoretic force. Namely, by the existence of the positive charge due to the excess charge on the hotter side of the metal, a negative charge is induced at the inside of the metal in the hotter region due to the image effect, whereas a positive charge is induced at the inside of the metal in the colder region because of the electric neutral condition. Then, the positive charge at the inside of the metal attracts negative ion on the colder side of the metal. Consequently, two kinds of electric double layers are formed at the hotter and colder sides of the metal. Then, the electric field $\left(E_{i e}\right)$ from the positive charge at the hotter side to the negative charge at the colder side is generated and it produces a quadrupolar vortex flow similar to ICEO, as shown in Fig. 12(a).

On the other hand, as shown in Fig. 12(b), SE-NTKP is driven by the excess positive ion that is pushed on the colder wall by the thermophoretic force. Namely, by the existence of the positive charge due to the excess charge on the colder wall, an electric field $\left(E_{s e(2)}\right)$ in the hotter direction is generated and it induces positive and negative charges at the inside of the metal in the hotter and colder regions. Then, similar to the ordinary ICEO, the positive and negative ions attract counter ions and form two kinds of electric double layers at the hotter and colder regions. However, different from the 
ordinary ICEO, the excess positive and negative ions that exist intrinsically near the hotter and colder sides due to the thermophoretic force weakens the zeta potential due to $E_{s e(1)}$ around the metal. Further, at the same time, the latent excess ions generate an electric field $\left(E_{s e(2)}=0.5 E_{i e}\right)$ and suppress the a tangential electric field outside of the double layer to the level of $E_{s e(1)}-E_{s e(2)}$. Furthermore, the intrinsic positive ion is pushed out to the left and right sides in the hotter region, whereas the intrinsic negative ion is pushed out to the left and right sides in the colder region, as partially shown in Fig. 9(d). Consequently, a 8-pole vortex flow is generated for Modes 5 and 6 , as shown in Fig. 12(b). Note that we observe an ordinary quadrupolar vortex flow for Mode 4 because of the difference of the early state and it results in the difference of the memory effect, as will be discussed later.

\subsection{AVF at the early stage}

The weak AVF is generated at the early stage for Mode 1 as shown in Fig. 2(a), whereas AVF is not generated for Mode 3 as shown in Fig. 3(a). This is because in Mode 1 , positive and negative space charges are generated at $x \sim 0.3$ and 0.7 , respectively, by the concentration polarization due to the charging current to the electric double layer of the metal. Similarly, by the thermophoretic flow near the wall, excess negative and positive space charges are generated at $x \sim 0.1$ and 0.9 , respectively and thus the electric field in the $-x$ direction is generated. Consequently, AVF is generated at the early stage. Further, the motion of the excess positive ion that forms positive charge region at the colder region is accelerated by the thermokinetic force, whereas the motion of the excess negative ion that forms negative charge region at the hotter region is suppressed by the thermokinetic force. Thus, AVF at the colder region is stronger than the hotter region.

However, in Mode 3, a strong electric field in the $-x$ direction is generated as shown in Fig. 10(d) because of the large temperature gradient near the wall at the early stage [in Fig. 3(f)], whereas the space charge cannot be generated around the particle because of the low temperature gradient near the particle at the early stage [in Fig. 3(f)]. Thus, AVF is not generated in Mode 3. Further, similar to the discussion of Mode 3, AVF is not generated in Mode 6 because of the lack of the space charge region around the particle as shown in Fig. 4(a).

\subsection{Response time and the strong peak in Modes 2 and 3}

Similar to the charging time of the electric double layer, we can define the characteristic time for the thermokinetic motion of the positive and negative ions as

$$
t_{ \pm}=\frac{\lambda_{D}^{2}}{2 D_{ \pm} \alpha_{ \pm}} .
$$

That is, $t_{+} / T_{0} \simeq 0.8 \times 10^{-5}$ and $t_{+} / T_{0} \simeq 1.0 \times 10^{-4}$ for the $\mathrm{HCl}$ solution. Here, $t_{+}$ corresponds to the peak time of $u_{\max }$ for Modes 2 and 3 in Figs. 3(d) and 8(a) and it is the time during which $\mathrm{H}^{+}$ion moves the distance of $\lambda_{D}$. Namely, since $\mathrm{Cl}^{-}$almost cannot move during $t_{+}$, the strong electric field $E_{c p}$ in the $-x$ direction [in Fig. 10(d)] occurs due to the concentration polarization owing to the strong flow of $\mathrm{H}^{+}$near walls resulting from a large temperature gradient in the early stage, whereas there is no space charge around the metal. Thus, we consider that $E_{c p}$ produces the strong vortex flow of $f=0.76$ based on the ordinary ICEO mechanism at $t \simeq t_{+} \simeq 10^{-5} T_{0}$ in Mode 2 and 3 in Figs. 3(d) and 8(a) and it decays as $E_{c p}$ becomes small because of the passing boundary condition. Further, $\mathrm{Cl}^{-}$moves the distance of $\lambda_{D}$ during $t_{-}$and thus the relaxation process is completed for Mode 1 at $t \simeq t_{-} \simeq 0.001 T_{0}$. 

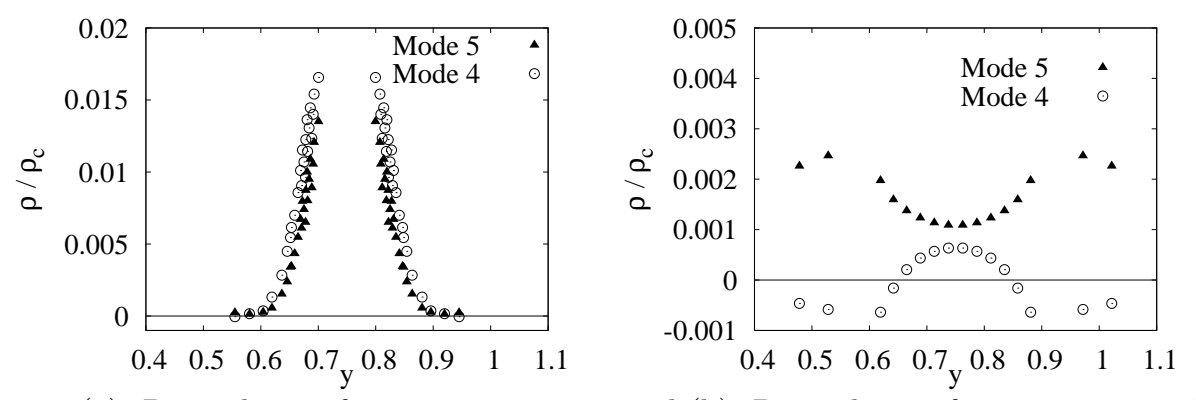

(a) Dependence of $\rho$ on $y$ at $x=0.58$ and (b) Dependence of $\rho$ on $y$ at $x=0.70$ and $t / T_{0}=2.0 \times 10^{-4}$ $t / T_{0}=2.0 \times 10^{-4}$
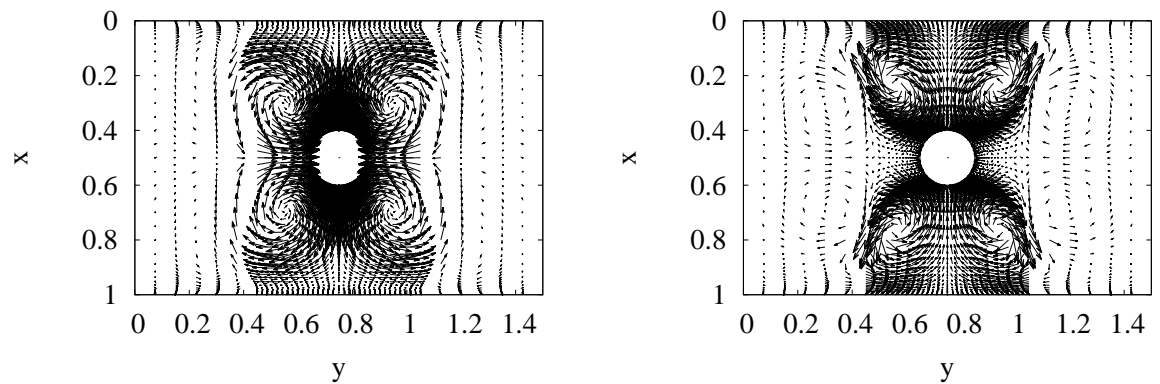

(c) AVF in Mode 4 at $t / T_{0}=2.0 \times 10^{-4}$ (d) $\operatorname{SVF}(8$ pole vortex flow) in Mode 5 at $\left(f^{\prime}=15\right)$

$t / T_{0}=2.0 \times 10^{-4}\left(f^{\prime}=15\right)$

FIGURE 13. Mechanism of the memory effect.

\subsection{Mechanism of the memory effect}

In Fig. 8(c), $u^{\max }$ of Mode 4 has a small peak at $t / T_{0}=10^{-4}$, whereas $u^{\max }$ of Mode 5 continues to glow and it reaches a quasi steady state at $t / T_{0} \simeq t_{-} \simeq 10^{-3}$. We consider that this branching is the origin of the memory effect. Namely, similar to the discussion in Secs. 4.7 and 4.8, the positive ion is much transported to the hotter side of the metal cylinder in Mode 4 than Mode 5 at the early stage $\left(t / T_{0}=2 \times 10^{-4}\right)$, as shown in Fig. 13(a). At the same time, the current causes negative charge regions around $(x, y) \simeq(0.7,0.6)$ and $(0.7,0.9)$ owing to the concentration polarization in Mode 4 , as shown in Fig. 13(b). Thus, in Mode 4, the positive charge around $(x, y) \simeq(0.7,0.75)$ moves to the $x$ direction and the negative charge around $(x, y) \simeq(0.7,0.6)$ and $(0.7,0.9)$ moves to the metal. Consequently, AVF is observed at $t / T_{0}=2 \times 10^{-4}$ for Mode 4 in Fig. 13(c). In this mode, the positive charge is transported to negative charge region, whereas the negative charge is transport to the positive region. Thus, the maximum positive charge at $x>0.5$ decreases rapidly in Mode 4, as shown in Fig. 9(b) and AVF changes to SVF as the sign of the charge density around $(x, y) \simeq(0.6,0.75)$ changes from positive to negative value.

Further, in Mode 5 , since the positive charge around $(x, y) \simeq(0.7,0.55)$ and $(0.7,0.95)$ is much larger than the positive charge around $(x, y) \simeq(0.7,0.75)$, SVF of the 8 pole type is observed at $t / T_{0}=2 \times 10^{-4}$, as shown in Fig. 13(d). In this mode, the positive charge is transported to the positive charge region. Thus, the maximum positive charge $\rho^{\max }$ at $x>0.5$ does not decrease rapidly in Mode 5, as shown in Fig. 9(b). We consider 
that the difference of the preserved maximum positive charge density at $x>0.5$ (the preserved maximum negative charge density at $x<0.5)$ in the laminate vortex flow in low Reynolds number is the main reason of the memory effect of NTKP. In addition, we consider that once the excess positive charge is pressed on the metal in the early stage [as shown in Fig. 13(a)], the effect is remained under the specific flows of Modes 4 and 5 because of the electrostatic attraction force between the image charge and the excess ion even if the other induced charge is generated by the Seebeck effect; i.e., we consider that the electrostatic attraction force due to the image effect is the other main reason of the memory effect of NTKP.

\subsection{Summary of the $1 D$ thermokinetic theory near the wall}

By the discussion of Sec. 4.1 and 4.2 with the Poisson equation we can write

$$
\rho=\rho_{w a l l} \mathrm{e}^{-\frac{X}{\lambda_{D}}}=-\varepsilon \frac{d^{2} \Phi}{d X^{2}}
$$

near the wall for the 1D thermokinetic problem under the blocking boundary condition. Thus, by integrating Eq. (4.29) from $X=X$ to $X=\infty$ under the condition that $\left(-\varepsilon \frac{d \Phi}{d X}\right)_{X=\infty}=S e(\nabla T)_{0}$, we obtain an electric field near the wall as

$$
E(X)\left(=-\frac{d \Phi}{d X}\right)=S e(\nabla T)_{0}-\frac{\rho_{\text {wall }} \lambda_{D}}{\varepsilon} \mathrm{e}^{-\frac{X}{\lambda_{D}}} .
$$

Therefor, by using $\sigma=\rho_{\text {wall }} \lambda_{D}=\varepsilon S e(\nabla t)_{0}$, we obtain

$$
E(0)=S e(\nabla T)_{0}-\frac{\rho_{w a l l} \lambda_{D}}{\varepsilon}=0 .
$$

That is, we can understand that the ion blocking boundary condition corresponds to the zero electric field condition, which is often used in this field; e.g., please see Refs. (Majee \& Würger 2012; Chikina et al. 2012, 2015). In fact, we find that $\frac{\partial \phi}{\partial x}=0$ at $x=0$ and 1 at $t / T_{0}=0.004$ in Fig. 11(d).

Further, by the discussion of Sec. 4.1 and 4.2 , we can write

$$
\begin{aligned}
C_{+} & \simeq C_{+0} \mathrm{e}^{-\frac{X}{l_{a}}}+C_{0}, \\
C_{-} \simeq C_{+}-\frac{\rho}{e z} & \simeq C_{+}-\frac{\rho_{0}}{e z} \mathrm{e}^{-\frac{X}{\lambda_{D}}}, \\
\frac{d C_{+}}{d X} \simeq-\frac{C_{+0}}{l_{a}} \mathrm{e}^{-\frac{X}{l_{a}}} & \simeq-2 \alpha_{+}(\nabla T)_{0} C_{0},
\end{aligned}
$$

where $l_{a}$ is an apparent thermal characteristic length and $C_{+0}=C_{+}-C_{0}$ at $X=0$. Although we obtain

$$
l_{a} \simeq \frac{C_{+0}}{2 \alpha_{+}(\nabla T)_{0}}
$$

$l_{a}$ is not universal value. Instead, it is defined as the distance, at which $\nabla C_{+}=\nabla C_{-}=0$ and thus $E(X)=$ const. Namely, since $\mathrm{e}^{-4} \simeq 0.02$, we can estimate

$$
l_{a} \sim 4 \lambda_{D}
$$

In other words, although the universal characteristic length of the system is $\lambda_{D}$, the apparent characteristic length exists. In fact, $\left(C_{+}-C_{0}\right) / C_{0}$ decays to $1 / e$ times of $C_{+0}$ at $X \sim 0.08 W=4 \lambda_{D}=4 \mu \mathrm{m}$ in Fig. 11(a). 


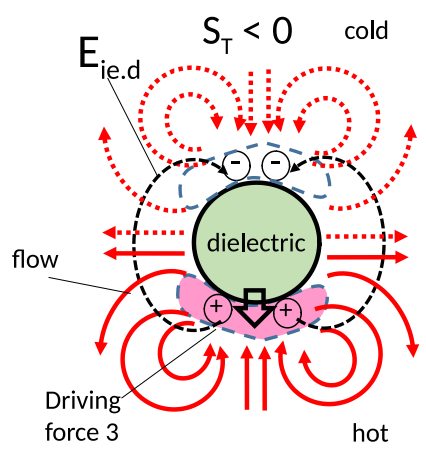

(a) DP-NTKP

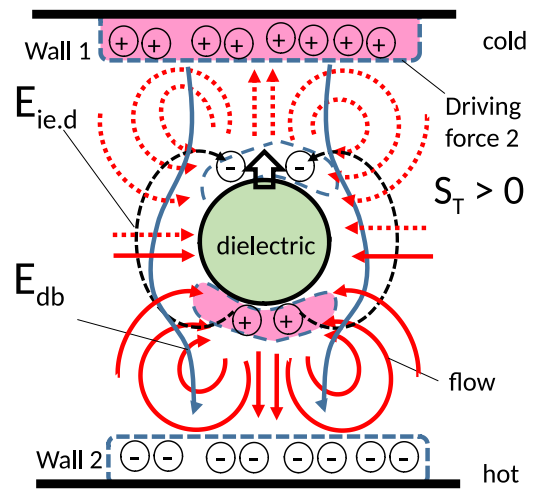

(b) DB-NTKP

FIGURE 14. (Color online) NTKP for a dielectric particle. (a) Dielectric-passing type NTKP (DP-NTKP). (b) Dielectric-blocking type NTKP (DB-NTKP).

\subsection{The effect of conductance of the particle on NTKP}

Interestingly, even when the particle (or cylinder) is made of dielectric material, we can predict that a similar kind of vortex flow is generated under the existence of the large temperature gradient, as shown in Fig. 14. That is, dielectric-passing type NTKP (DPNTKP) and dielectric-blocking type NTKP (DB-NTKP) exist for the dielectric particle. That is, similar to IE-NTKP, the swifter positive ion is pressed on the hotter side of the particle under the passing ion boundary condition and thus $C_{+}$increases at the hotter side due to the thermophoretic force, whereas $C_{+}$decreases at the colder side of the particle. Thus, a vortex flow similar to IE-NTKP is generated for DP-NTKP, as shown in Fig. 14(a). However, different from the particle of the metal, we cannot expect the focusing effect of temperature described by Eq. (4.6) and the image effect of the metal. Thus, the zeta potential of the dielectric particle $\zeta_{i e . d}^{\prime \prime}$ and the driving electric field of the dielectric particle $E_{i e . d}$ become $1 / 8$ times of those of metal; i.e., $\zeta_{i e . d}^{\prime \prime}=\frac{1}{8} \zeta_{i e}^{\prime \prime}$ and the driving electric field of the dielectric particle $E_{i e . d}=\frac{1}{8} E_{i e}$. Therefore, the maximum flow velocity $u_{\text {passing }}^{\text {dielectric }} \simeq \frac{1}{64} u_{i e}$. That is, we obtain the observable maximum flow velocity around the dielectric particle for the passing ion boundary condition as

$$
\begin{array}{r}
u_{d p} \simeq \frac{\varepsilon \zeta_{i e . d}^{\prime \prime}}{2 \mu} E_{i e . d}, \\
\zeta_{i e . d}^{\prime \prime} \simeq \sqrt{2} \alpha_{+}\left(\nabla_{n} \tilde{T}\right)_{0} \frac{\lambda_{D}^{3} \rho_{c}}{\varepsilon W}, \\
E_{i e . d} \simeq-\frac{4 \alpha_{+}\left(\nabla_{n} \tilde{T}\right)_{0} \frac{\lambda_{D}^{3} \rho_{c}}{\varepsilon W}}{\pi\left(a+\lambda_{D}\right)} .
\end{array}
$$

Further, similar to SE-NTKP, the swifter positive ion is pressed on the colder side of the wall under the blocking ion boundary condition and thus $C_{+}$increases at the colder wall due to the thermophoretic force, whereas $C_{+}$decreases at the hotter wall. However, 


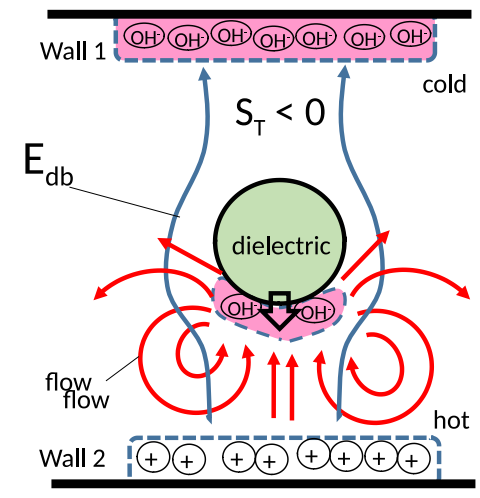

(a) DB-NTKP in $\mathrm{NaOH}$ solution

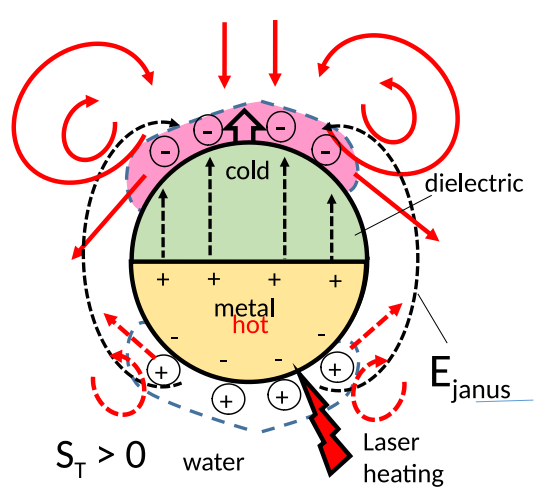

(b) NTKP on Janus particle

FiguRE 15. (Color online) Relevant experiments on NTKP.

different from the particle of the metal, the excess swifter positive ion still exists on the hotter side of the particle and the shortage of the swifter positive ion exists at the colder side, since the image (or induced charge) effect of a dielectric particle is almost negligible compare to metal. Thus, the positive and negative charges on the hotter and colder sides of the particle move by the Seebeck electric field due to the charge near the wall. Therefore, a quadrupolar but asymmetrical vortex flow is generated around the dielectric particle for DB-NTKP under the blocking boundary condition, as shown in Fig. 14(b). Further, similar to the discussion of Eqs. (4.11) and (4.5), we obtain the observable maximum flow velocity around the dielectric particle for the blocking ion boundary condition as

$$
\begin{array}{r}
u_{d b} \simeq \frac{\varepsilon \zeta_{d b}^{\prime \prime}}{2 \mu} E_{d b}, \\
\zeta_{d b}^{\prime \prime} \simeq \sqrt{2} \alpha_{+}\left(\nabla_{n} \tilde{T}\right)_{0} \frac{\lambda_{D}^{3} \rho_{c}}{\varepsilon W}, \\
E_{d b} \simeq 2 E_{s e(1)} \sin \frac{\pi}{4} \simeq 2 \sqrt{2} \alpha_{+}\left(\nabla_{n} \tilde{T}\right)_{0} \frac{\lambda_{D}^{2} \rho_{c}}{\varepsilon W},
\end{array}
$$

where we assume that the effect of $E_{i e . d}$ is negligible for the dielectric particle.

Note that we consider that the charge of the hotter side is negligible in DP- and DB-NTKP because of the continuous instant supply of the positive ion from the hotter region and thus the vortex flow at the colder side is negligible, as expressed by the broken lines in Fig. 13. Further, by using Eqs. (4.37) to (4.42), we obtain $E_{d b} \simeq 350$ $\mathrm{V} / \mathrm{m}, \zeta_{d b}^{\prime \prime} \simeq 0.17 \mathrm{mV}$, and $u_{d b} \simeq 0.021 \mu \mathrm{m} / \mathrm{s}$ in DB-NTKP; $E_{d p} \simeq 26 \mathrm{~V} / \mathrm{m}, \zeta_{d p}^{\prime \prime} \simeq 0.17$ $\mathrm{mV}$, and $u_{d p} \simeq 0.0016 \mu \mathrm{m} / \mathrm{s}$ in DP-NTKP. Thus, we might have a chance to observe DB-NTKP, although DP-NTKP might be difficult to observe. 


\subsection{Relevant experiments}

Discussion of DB-NTKP provides a simple theory for the thermophoresis (or Soret effect) of the electrically neutral dielectric particle, although a thermophoretic theory for the charged colloids was provided by Würger (Würger 2008). Namely, the thermophoretic velocity $U$ of the dielectric particle is provided as

$$
U_{t h . d} \sim u_{d b} \sim \frac{\varepsilon \lambda_{D}}{2 \mu}\left[2 \alpha_{+}\left(\nabla_{n} \tilde{T}\right)_{0}\right]^{2}\left(\frac{\lambda_{D}^{2} \rho_{c}}{\varepsilon W}\right)^{2} \sim \frac{\varepsilon \lambda_{D}}{2 \mu}\left[S e(\nabla T)_{0}\right]^{2}
$$

where $\frac{\lambda_{D}^{2} \rho_{c}}{\varepsilon W}=\frac{1}{2} \Phi_{c} \simeq 12.9 \mathrm{mV}$. The surprising aspect of Eq. (4.43) is that the Soret effect due to the ion that is thermokinetically pressed on the particle is considered to be a kind of NTKP since $u_{t h . d} \propto(\nabla T)_{0}^{2}$; thus, $D_{T}$ and $S_{T}$ are proportional to $\nabla T$. In addition, our theory predicts that the dielectric particle moves toward the colder region when the thermokinetically swifter ion is a positive ion (e.g., $\mathrm{H}^{+}$) in the electrolyte as shown in Fig. 14(b), whereas the dielectric particle moves toward the hotter region when the thermokinetically swifter ion is a negative ion (e.g., $\left.\mathrm{OH}^{-}\right)$in the electrolyte as shown in Fig. 15(a); this prediction agrees with the experimental results of Vigolo et al. (Vigolo et al. 2010). Further, our theory predicts that $u_{t h . d}$ decreases as $C_{0}$ increases through $\lambda_{D}$ and this prediction also agrees with the experimental results of Vigolo et al. (Vigolo et al. 2010).

Further, we consider that the self-propulsion motion of the Janus particle irradiated by the laser (Jiang et al. 2010) can be explained by NTKP theory, since the experimental thermokinetic velocity of the self-propelled Janus particle $u_{J}$ (Jiang et al. 2010) does not proportional to $\nabla T$ globally in the sense that the line of the dependence $\left(u_{J}\right.$ vs. $\nabla T)$ does not go through zero point, as mentioned before. Namely, since $\alpha_{+}^{H^{+}}=2.7$ and $\alpha_{-}^{O H^{-}}=3.4$ in water, the swifter negative ion is pressed on the colder dielectric surface, whereas the slower positive ion remains on the colder dielectric surface, as illustrated in Fig. 15(b). Then, negative induced charge is generated on the metal surface, whereas positive induced charge is generated at the interface between metal and dielectric material and it attracts negative ion toward the metal region. Consequently, the strong surface flow toward the metal region causes on the dielectric surface. Thus, the Janus particle moves toward the dielectric end in water. Further, by the similar mechanism, the Janus particle moves toward the metal end in a Triton X-solution because of the decreasing of $\mathrm{pH}$ due to the effect of the hydroxy group of the Triton X-solution. This explanation agrees with the experimental results (Jiang et al. 2010) and thus our theory justified to some extent.

\subsection{D model and the general picture on NTKP}

Through the discussion of the previous sections, we recognized that NTKP is a general phenomenon that can be found in both metal and dielectric particle. That is, the original driving force of NTKP is the excess ions pressed on the particle by the thermokinetic force due to the thermal gradient and the flow around the particle is generated by the interaction between the excess ion and the electric field that is made by the excess ion itself and/or the Seebeck electric fields due to the blocking boundary condition on the wall of the liquid chamber; thus, the flow velocity is intrinsically proportional to the square of the temperature gradient. The difference between the metal NTKP and the dielectric NTKP is the strength of the polarization of the particle resulting from the permittivity $\varepsilon$ and the strength of the local temperature gradient of the particle resulting from the thermal conductivity $\lambda$. Namely, by the difference of $\varepsilon$ and $\lambda$, the flow velocity of the metal NTKP is approximately 8 times of that of the dielectric NTKP. 
Here, we consider a 3D model using Debye-Hükel approximation for the thick electric double layer (i.e., $\lambda_{D} \gg a$ ). That is, for the $3 \mathrm{D}$ dielectric particle, we can approximate that $\left(\pi a^{2} \varepsilon S e \nabla T\right) E_{\text {interact }} \simeq 6 \mu U a$ for the blocking boundary condition, where $q \simeq$ $\varepsilon S e \nabla T$ is a charge of the swifter excess ion on the hotter side, $U$ is the velocity of the particle, and $E_{\text {interact }}$ is an electric field that interacts with the charge around the particle;i.e., we obtain that

$$
U_{\text {dielectric }}^{\lambda_{D} \gg a} \sim \frac{\varepsilon a}{6 \mu}(S e \nabla T) E_{\text {interact }} .
$$

Further, by multiplying $\frac{3}{2}$ factor to Eq. (4.44), we obtain $U$ for the thin electric double layer (i.e., $\left.\lambda_{D} \ll a\right)$; i.e.,

$$
U_{\text {dielectric }}^{\lambda_{D} \ll a} \sim \frac{\varepsilon a}{4 \mu}(S e \nabla T) E_{\text {interact }} .
$$

Although $U$ is generally depend on the difference of the velocities of the vortex flows on the hotter and colder sides of the particle, $u^{\max }$ is generally described as

$$
u_{\text {general }}^{\max } \sim f_{p} f_{\kappa} \frac{\varepsilon a}{4 \mu}(S e \nabla T) E_{\text {interact }},
$$

where $f_{p}$ is the figure of merit due to $\varepsilon$ and $\lambda$ and $f_{\kappa}$ is the factor due to $\kappa=a / \lambda_{D}$. Note that $f_{p} \simeq 8$ for the metal and $f_{p} \simeq 1$ for the dielectric particle; $f_{\kappa} \simeq 1$ at $\kappa=\infty$ and $f_{\kappa} \simeq \frac{2}{3}$ at $\kappa=0 ; E_{\text {interact }} \sim S e \nabla T$.

\subsection{The influence of the electrolyte concentration on IE- and SE-NTKP}

From Eqs. (4.16) and (4.28), the maximum flow velocities of IE- and SE-NTKP depend on the concentration since $\lambda_{D} \propto 1 / \sqrt{C_{0}}$ and $\rho_{c}=e z C_{0}$. In detail, $u_{i e} \propto 1 / C_{0}$ and thus $u_{i e} \rightarrow 0$ at $C_{0} \rightarrow \infty$ in IE-NTKP. Further, $u_{s e}$ increases as $C_{0}$ increase in SE-NTKP. This is because $E_{s e}$ increases as $C_{0}$ increases, since $E_{s e(2)} \propto 1 / \sqrt{C_{0}}$ and $E_{s e(1)}$ is constant. In addition, $\left|\zeta_{s e}^{\prime \prime}\right|$ increases as $C_{0}$ increases, since $\rho_{\text {se.m(2) }} \frac{\lambda_{D}^{2}}{\varepsilon} \propto 1 / \sqrt{C_{0}}$ and $\rho_{\text {se.m(1) }} \frac{\lambda_{D}^{2}}{\varepsilon}$ is constant. Thus, at $C_{0} \rightarrow \infty$, we obtain

$$
u_{s e} \rightarrow-\frac{\varepsilon a}{\mu}\left[2 \alpha_{+}\left(\nabla_{n} \tilde{T}\right)_{0}\right]^{2}\left(\frac{\lambda_{D}^{2} \rho_{c}}{\varepsilon W}\right)^{2}
$$

and it is consistent with Eq. (4.46). Namely, as a whole, our new theories on IE-, SE-, DP-, and DB-NTKP are consistent with each other and they explain various numerical results under the various conditions reasonably, as discussed in the previous sections. Thus, our new theories are useful. Nevertheless, we may need to develop more precise theory in the future, since Eq. (4.46) suggest that $u_{i e}=$ const. for various $C_{0}$. In addition, we may need to reconsider " $S e^{\prime \prime}$ relating to NTKP in the future since $E_{\text {interact }}$ may change continuously from zero to Se defined by Eq. (2.24) for the passing boundary condition; e.g., $E_{\text {interact }}$ on a vertical plate between the hotter and colder electrodes is provably small since there is no pressed excess ion on the wall, whereas $E_{\text {interact }}$ on a horizontal plate between the hotter and colder electrodes is provably near to Se of Eq. (2.24).

\subsection{Convection flow}

We neglect the convection flow for our calculation. This is because at least theoretically, it is prevented when the hotter wall is placed at the higher place horizontally. Moreover, Rayleigh number $R a \equiv \frac{g \beta \Delta T W^{3}}{\nu a_{T}} \simeq 2.29 \times 10^{-14} \ll 1$, Marangoni number $|M a| \equiv$ $\frac{\frac{\partial \sigma}{\partial T} \Delta T W}{\mu a_{T}} \simeq 0.42 \times 10^{-3} \ll 1$, and $R a /|M a|=\frac{g \frac{\partial \rho}{\partial T} W^{2}}{\frac{\partial \sigma}{\partial T}} \simeq 5.5 \times 10^{-11} \ll 1$, where $\sigma$ is 
a surface tension, $\mu=0.668 \mathrm{~m}^{2} / \mathrm{s}$ is a kinematic viscosity, $\beta=-\frac{1}{\rho} \frac{\partial \rho}{\partial T}=0.38 \times 10^{-3}$ is a coefficient of thermal expansion, $\Delta T=50 \mathrm{~K}, W=50 \mu \mathrm{m}, \Delta \sigma=8.26 \mathrm{mN} / \mathrm{m}$, $\mu=0.653 \mathrm{mPa} \mathrm{s}, a_{T}=1.52 \mathrm{~m}^{2} / \mathrm{s}$, and $g=9.8 \mathrm{~m} / \mathrm{s}^{2}$. Thus, in our system, Marangoni convection (due to the difference of the surface tension) and Rayleigh convection (due to the difference of the density) are suppressed because of the viscous force. In fact, the convection flow is usually suppressed in the experiment in this field (Bonetti et al. 2011; by Paul Delahay 1963). For example, Bonetti et al. (Bonetti et al. 2011) prevent a convection flow and reported huge Seebeck coefficients in nonaqueous electrolytes by using a Teflon hollow cylinder (15 mm high) sealed by $5 \mathrm{~mm}$ thick sapphire window plates and setting the hotter sapphire plate $\left(T=28\right.$ to $\left.75{ }^{\circ} \mathrm{C}\right)$ at the higher position and the colder sapphire plate $\left(T=24^{\circ} \mathrm{C}\right)$ at the lower position; i.e., experimentally a convection flow can be suppressed significantly under the condition of the temperature difference of 4 to $51 \mathrm{~K}$ over the gap distance of $15 \mathrm{~mm}$. Thus, to neglect a convection flow in our simulation is justified. Note that the vertically sandwiched configuration that places the hotter plate at the higher position is useful to minimize a natural convection flow since convection flow occurs by the upward motion of the lower density solution at the lower position. Further, the sapphire plates of the large thermal conductivity were used to establish a uniform heating of the sample cross section and a stable temperature gradient.

\subsection{The effect of the temperature dependence of $D_{ \pm}$and $\mu$}

In Fig. 5, the effect of the temperature dependence of $D_{ \pm}$and $\mu$ is rather small. This is because Eq. (2.3) already includes the main temperature dependence of parameters even if we assume that $D_{ \pm}$and $\mu$ are constant. Further, the NTKP characteristic is mainly governed in the region of an electric double layer of the thickness $\lambda_{D}(\simeq 1 \mu \mathrm{m})$ and the surface temperature of the metal cylinder is assumed to be constant; i.e., the dominant temperature difference is considered as $\Delta T^{\text {dominant, ntkp }} \simeq 2 \lambda_{D}(\nabla T)_{0}=2 \mathrm{~K}$ and thus the effect of the temperature dependence of the parameters is small. Note that the dominant temperature difference for the ordinary linear thermokinetic phenomenon (LTKP) is usually considered as $\Delta T^{\text {dominant }, l t k p} \simeq 2 a(\nabla T)_{0}=10 \mathrm{~K}$ since the thermal conductivity of a nonpolarizable particle is assumed to be compatible with that of the surrounding solution. Thus, the effect of the temperature dependence of the parameters of the NTKP is much smaller than that of the ordinary thermokinetic phenomena.

\subsection{Physical meaning of the comparative study of the various modes}

By comparing the numerical results for the passing and blocking boundary conditions, we first recognized the precise physical picture of NTKP. Thus, those boundary conditions are needed for our analysis. Specifically only blocking boundary condition (or only zero electric field condition) is usually considered at the wall in the field of thermokinetics, although the researchers for a thermogalvanic cell might be interested in the passing boundary condition. However, since SE-NTKP includes the effect of IE-NTKP, the blocking boundary condition is required to clarify IE-NTKP. Further, the passing and blocking boundary conditions are known as major boundary conditions to understand the phenomena in the field of electrokinetics. Thus, even in the field of the thermokinetic phenomena, we consider that the passing boundary condition will be important in the future.

Further, by comparing the numerical results for the different initial conditions on the temperature distribution, we find that the memory effect (or hysteresis effect of the temperature) exists in SE-NTKP. Specifically, we consider Eq. (2.1) as the limit of 
$a_{T} \rightarrow \infty$ for Modes 1 and 4 and the suddenly applied hot and cold temperature at the walls for Modes 2 and 5. Here, the suddenly applied hot and cold temperature is an idealized condition as the limit of the instant heating and cooling of walls. However, at least the instant heating can be realized to some extent experimentally by using a thin film heater in the water and thus the situation similar to Modes 2 and 5 occurs experimentally. Thus, the initial conditions for temperature are useful to understand nonsteady phenomena, as a first step.

In addition, since we consider the temperature difference $\Delta T$ is $50 \mathrm{~K}$ across the chamber, the variation of the coefficients of viscosity, mobility, and diffusion is not negligible as the general (but too strict) argument. However, the consideration of the temperature dependences of those parameter complicates the intrinsic phenomena in NTKP. Thus, as a challenging study, we calculates both situations. Through this timeconsuming calculations, we first succeed in clarifying NTKP and answering to the general criticism, although the effect of the temperature dependence is reasonably small as a result, as discussed in Sec. 4.16 .

\section{Conclusion}

In conclusion, we clarified the general physical picture on NTKP. That is, by performing a nonsteady multi-physics direct simulation, we have shown that (1) the maximum flow velocity around the metal is generally proportional to the square of the temperature gradient. (2) NTKP is classified two types by their mechanism; i.e., IE-NTKP for the passing boundary condition and SE-NTKP for the blocking boundary condition. (3) The memory effect appears in SE-NTKP by the difference of the temperature gradient at the early stage. (4) The strong peak of $u^{\max }$ appears at the early stage for the passing boundary condition. (5) The temperature dependence effect of the parameters is rather small but detectable. Further, through the discussion, (6) new theories on IENTKP and SE-NTKP are provided and they explain the numerical results in a selfconsistent manner. (7) AVF is explained by the concentration polarization due to the rapid transportation of the swifter ion in the early stage. (8) Response time is explained by the thermokinetic diffusion time; i.e., $t_{ \pm}=\lambda_{D}^{2} / 2 D_{ \pm} \alpha_{ \pm}$. (9) The memory effect in SE-NTKP (in particular Mode 5) is qualitatively explained by the attractive force due to the image effect and the preserving effect of the initial charge due to the 8 pole vortex flow. (10) A simple 1D thermokinetic theory is provided and it shows that the blocking boundary condition leads the zero electric field condition and $\lambda_{D}$ is the only true characteristic length for our system, although the apparent thermal characteristic length $\left(l_{a} \sim 4 \lambda_{D}\right)$ exists. (11) A theory on NTKP for the dielectric particle is provided and it seems to explain the Soret effect of a neutral dielectric particle to some extent. (12) Self propelled motion of the Janus particle irradiated by a laser is successfully explained as the framework of NTKP theory. (13) A simple 3D model on NTKP is provided and it clarifies NTKP for the general particle. We believe that our findings give a new insight on the better understanding of the general thermokinetic phenomena ranging from a metallic particle to a dielectric particle. In particular, the most surprising aspect of our findings is that there is possibility that NTKP exists universally. Further, similar to ICEO, the 3D NTKP effects of the 3D particles or structures (e.g., the transportation effect due to the broken symmetry, the rotation effect of an ellipsoid particle, the connecting effect due to the hydrodynamic interaction between particles, etc.) will be explored with promising applications in the future.

This work was partially supported by JSPS KAKENHI Grant Number JP16K05650. 


\section{REFERENCES}

Agar, J. N., Mou, C. Y. \& LONG Lin, Jeong 1989 Single-ion heat of transport in electrolyte solutions. a hydrodynamic theory. J. Phys. Chem. 93, 2079.

Bazant, M. Z., Kilic, M. S., Storey, B. D. \& Ajdari, A. 2009 Towards an understanding of induced-charge electrokinetics at large applied voltages in concentrated solutions. Adv. Colloid Interf. Sci. 152, 48.

Bazant, M. Z. \& Squires, T. M. 2004 Induced-charge electrokinetic phenomena: Theory and microfluidic applications. Phys. Rev. Lett. 92 (3), 066101.

Bickel, Thomas, Majee, Arghya \& Würger, Alois 2013 Flow pattern in the vicinity of self-propelling hot janus particles. Phys. Rev. E 88, 012301.

Bonetti, M., Nakamae, S., Roger, M. \& Guenoun, P. 2011 Huge seebeck coefficients in nanoaqueous electrolytes. J. Chem. Phys. 134, 114513.

E. N. Da C. Andrade, F. R. S 1952 Viscosity of liquids. Proceedings of the Royal Society of London A: Mathematical, Physical and Engineering Sciences 215, 36-43.

Chikina, I., Shikin, V. \& Varlamov, A. A. 2012 Seebeck effect in electrolytes. Phys. Rev. E 86, 011505.

Chikina, I., Shikin, V. B. \& Varlamov, A. A. 2015 Effect of boundary conditions on the character of ambipolar diffusion in electrolytes. Phys. Rev. E 92, 012310.

Davidson, Scott M., Andersen, Mathias B. \& Mani, Ali 2014 Chaotic induced-charge electro-osmosis. Phys. Rev. Lett. 112, 128302.

Duhr, S. \& Braun, D. $2006 a$ Geometry of reaction interfaces in chaotic flows. Phys. Rev. Lett. 97, 038103.

Duhr, Stefan \& Braun, Dieter 2006b Thermophoretic depletion follows boltzmann distribution. Phys. Rev. Lett. 96, 168301.

Eastman, E. 1926 Thermodynamics of non-isothermal systems. J. Am. Chem. Soc. 48, 1482.

Eastman, E. 1928 Theory of the soret effect. J. Am. Chem. Soc. 50, 283.

Eslamian, Morteza \& SAghir, M. Ziad 2009 Microscopic study and modeling of thermodiffusion in binary associating mixtures. Phys. Rev. E 80, 061201.

Gangwal, S., Cayre, O. J., Bazant, M. Z. \& Velev, O.D. 2008 Induced-charge electrophoresis of metallodielectric particles. Phys. Rev. Lett. 100, 058302.

GIDDings, J. 1993 Field-flow fractionation: Analysis of macromolecular, colloidal, particulate materials. Science 260, 1456.

Giddings, J., Shinude, P. \& Semenov, S. 1995 Thermophoresis of metal particles in a liquid. J. Colloid and interface science 176, 454.

Gregersen, Misha Marie, Andersen, Mathias Bækbo, Soni, Gaurav, Meinhart, Carl \& Bruus, HENRIK 2009 Numerical analysis of finite debye-length effects in induced-charge electro-osmosis. Phys. Rev. E 79, 066316.

Gutherie, G., Wilson, J. N. \& Schomaker, V. 1949 Theory of the thermal diffusion of electrolytes in a clusius column. J. Chem. Phys. 17, 310.

Hu, Renchong, Cola, Baratunde A., Haram, Nanda, Barisci, Joseph N., Lee, Sergey, Stoughton, Stephanie, Wallace, Gordon, Too, Chee, Thomas, Michael, Gestos, Adrian, Cruz, Marilou E. dela, Ferraris, John P., Zakhidov, Anvar A. \& BAUGHMAN, RAY H. 2010 Harvesting waste thermal energy using a carbon-nanotubebased thermo-electrochemical cell. Nano Letters 10 (3), 838-846.

Huang, F., Chakraborty, Lundstrom, C. C., Holmden, C., Glessner, J. J. G., Kieffer, S. W. \& LESHER, C. E. 2010 Isotope fractionation in silicate melts by thermal diffusion. Nature 464, 396.

Jiang, H.-R., Yoshinaga, N. \& Sano, M. 2010 Active motion of a janus particle by selfthermophoresis in a defocused laser beam. Phys. Rev. Lett. 105, 268302.

JSME 2009 JSME Data Book: Heat Transfer 5th Edition. JSME.

LI, Y. \& Gregory, S. 1974 Diffusion of ions in sea water and in deep-sea sediments. Geochimica et Cosmochica Acta 88, 708.

Majee, A. \& Würger, A. 2012 Charging of heated particles using the electrolyte seebeck effect. Phys. Rev. Lett. 108 (11), 118301.

Pascall, Andrew J. \& Squires, Todd M. 2010 Induced charge electro-osmosis over controllably contaminated electrodes. Phys. Rev. Lett. 104, 088301. 
By Paul Delahay, Edited 1963 J. N. Agar, Thermogalvanic Cells. in "Advances in Electrochemistry and Electrochemical Engineering", vol. 3, Chapter. 2. Interscience, New York.

Putnam, Shawn A. \& Cahill, David G. 2005 Transport of nanoscale latex spheres in a temperature gradient. Langmuir 21 (12), 5317-5323.

Putnam, Shawn A., Cahill, David G. \& Wong, Gerard C. L. 2007 Temperature dependence of thermodiffusion in aqueous suspensions of charged nanoparticles. Langmuir 23 (18), 9221-9228.

Ramos, A., Gonzalez, A., Castellanos, A., Green, N. G. \& Morgan, H. 2003 Pumping of liquids with ac voltages applied to asymmetric pairs of microelectrodes. Phys. Rev. E 67, 056302 .

Ramos, A., Morgan, H., Green, N. G. \& Castellanos, A. 1999 Ac electric-field-induced fluid flow in microelectrodes. J. Colloid Interf. Sci. 217, 420.

Squires, T. M. 2009 Induced-charge electro-kinetics: fundamental challenges and opportunities. Lab. Chip 9, 2477.

Squires, T. M. \& BazAnt, M. Z. 2004 Induced-charge electro-osmosis. J. Fluid Mech. 509, 217.

Sugioka, H. 2010 Chaotic mixer using electro-osmosis at finite peclet number. Phys. Rev. E 81, 036306.

SugioKA, HidEYUKI 2011 Basic analysis of induced-charge electrophoresis using the boundary element method. Colloids and Surfaces A: Physicochemical and Engineering Aspects 376 (1), $102-110$.

SugiokA, H. 2014a dc step response of induced-charge electro-osmosis between parallel electrodes at lage voltages. Phys. Rev. E 90, 013007.

SugiokA, H. $2014 b$ Nonlinear thermokinetic phenomena due to the seebeck effect. Langmuir 30, 8621 .

Sugioka, Hideyuki 2015 a Elastic valve using induced-charge electro-osmosis. Phys. Rev. Applied 3, 064001.

Sugioka, Hideyuki $2015 b$ Strategies on improving the micro-fluidic devices using the nonlinear electro- and thermo-kinetic phenomena. Advances in Colloid and Interface Science 226, Part A, $44-53$, colloid and Polymer Interfaces in Bio-resources and Environments.

Sugioka, Hideyuki 2016 Direct simulation of phase delay effects on induced-charge electroosmosis under large ac electric fields. Phys. Rev. E 94, 022609.

Urbanski, J. P., Thorsen, T., Levian, J. A. \& Bazant, M. Z. 2006 Fast ac electro-osmotic micropumps with nonplanar electrodes. Appl. Phys. Lett. 89, 143508.

Vigolo, Daniele, Buzzaccaro, Stefano \& Piazza, Roberto 2010 Thermophoresis and thermoelectricity in surfactant solutions. Langmuir 26 (11), 7792-7801.

WÜRGER, A. 2007 Thermophoresis in colloidal suspensions driven by marangoni forces. Phys. Rev. Lett. 98 (13), 138301.

WÜrger, A. 2008 Transport in charged colloids driven by thermoelectricity. Phys. Rev. Lett. 101, 108302.

WÜrger, A. 2010 Thermal non-equlibrium transport in colloids. Rep. Prog. Phys. 73, 126601. 